\title{
JOINT QUEUE-PERTURBED AND WEAKLY COUPLED POWER CONTROL FOR WIRELESS BACKBONE NETWORKS
}

\author{
Thomas Otieno OLWAL ${ }^{*, * *}$, KARIM DJOUANI ${ }^{*, * *}$, OKUThe P. KOGEDA*, \\ BAREND JACOBUS VAN WYK* \\ * Council for Scientific and Industrial Research (CSIR) \\ Tshwane University of Technology, Box 395, Pretoria 0001, South Africa \\ e-mail: \{thomas.olwal, kogeda\} @gmail.com, vanwykb@tut.ac. za \\ ${ }^{* *}$ University of Paris-Est \\ Aile Vicat bureau V426, 6-8 avenue Blaise Pascal, 77454 Marne la Vallee cedex 2, Creteil, France \\ e-mail: djouani@ieee.org
}

\begin{abstract}
Wireless Backbone Networks (WBNs) equipped with Multi-Radio Multi-Channel (MRMC) configurations do experience power control problems such as the inter-channel and co-channel interference, high energy consumption at multiple queues and unscalable network connectivity. Such network problems can be conveniently modelled using the theory of queue perturbation in the multiple queue systems and also as a weak coupling in a multiple channel wireless network. Consequently, this paper proposes a queue perturbation and weakly coupled based power control approach for WBNs. The ultimate objectives are to increase energy efficiency and the overall network capacity. In order to achieve this objective, a Markov chain model is first presented to describe the behaviour of the steady state probability distribution of the queue energy and buffer states. The singular perturbation parameter is approximated from the coefficients of the Taylor series expansion of the probability distribution. The impact of such queue perturbations on the transmission probability, given some transmission power values, is also analysed. Secondly, the inter-channel interference is modelled as a weakly coupled wireless system. Thirdly, Nash differential games are applied to derive optimal power control signals for each user subject to power constraints at each node. Finally, analytical models and numerical examples show the efficacy of the proposed model in solving power control problems in WBNs.
\end{abstract}

Keywords: decentralized power control, singular perturbation theory, weak coupling theory, wireless backbone networks, optimal control theory.

\section{Introduction}

In wireless networks, backbone networks are typically composed of stationary nodes that convey a large amount of traffic generated by wireless clients to a few nodes that act as gateways to the Internet (Bruno et al., 2005). In order to achieve this goal, Wireless Backbone Networks (WBNs) need to be fully distributed in both structure and function. They should also be reliable in terms of energy efficiency and robustness against the adverse wireless link conditions. Furthermore, in order to meet high traffic demands, WBNs may be equipped with multiple radios or Network Interface Cards (NICs) and/or operate on the multiple orthogonal frequency channels (Engim, 2004). In this scenario, a backbone node has a radio with its own Medium Access Control (MAC) and physical layers (PHY). Thus, a single wireless Multi-Radio MultiChannel (MRMC) node may access the client network and route the backbone traffic simultaneously. An additional requirement is that such networks should be selfmanaging and can form high capacity Wireless Mesh Networking (WMN) with packet stripping capabilities (Zhu et al., 2008; Adisehu et al., 1996).

The operation of such wireless MRMC backbone networks generally requires sustainable and reliable energy supplies. However, such networks are now being deployed in energy-constrained geographical regions where electric power outlets are scarce and alternative sources are the main energy amenities (Ishmael et al., 2008). In addition, power control problems including the inter (or intra) channel interference, unscalable connectivity and high energy depletion subsequently become prevalent. 
Therefore, the problem facing each wireless transmitreceive NIC or node pair (i.e., user) is how to regulate its transmission power level so that it is high enough to reach the intended receiver (i.e., for decoding a signal and maintaining connectivity) and low enough to maintain a minimal interference against neighbouring nodes and minimal energy consumption.

Numerous works have addressed the power control problem in distributed wireless networks with single radio configurations (Ramamurthi et al., 2008; Li and Hou 2004; 2005; Tsheng et al., 2001; Sorooshyari and Gajic, 2008; Arora and Krunz, 2007; Klues et al., 2006; Sheth and Han, 2005). The main goal is to resolve topology issues (Li and Hou 2004; 2005; Chen et al., 2007), cochannel interference (Tsheng et al., 2001; Sorooshyari and Gajic, 2008; Wang et al., 2006; Arora and Krunz, 2007) and energy-consumption (Klues et al., 2006; Sheth and Han, 2005). However, such techniques only solve the client access network power control problems where there are no multiple radios installed. Multiple radio configurations present additional power control challenges (Zhu et al., 2008). Recent research attempts have addressed the power control problems in wireless MRMC configurations (Chen et al., 2007; Olwal et al., 2009a; $2009 b ; 2009$ c). For instance, an energy-efficient Power selection Multi-radio Multi-channel Unification Protocol (PMMUP) has been proposed at the link layer to coordinate independent operations of multiple radios operating in separate wireless channels (Olwal et al., 2009a; 2009b; 2009c). Such approaches simplify the complexity involved with the multiple MAC and PHY layers and also ensure that the network connectivity stays scalable (Adisehu et al., 1996).

However, such approaches do not tackle transmission energy consumption problems due to perturbations of multiple-queues system (Avrachenkov, 1999). A queue perturbation arises when wireless channel links cause packet losses, hence a need for packet retransmissions at the expense of additional transmission energy. Furthermore, multiple independent and active collocated radios do interfere significantly with each other due to the orthogonality violation of wireless links. This is the problem of weakly coupled wireless control systems (Gajic and Shen, 1993). Investigating the effects of singular perturbation phenomena (in which the rate of energy consumption and queue evolution differ) and weak coupling of multiple channels on power control in wireless network is the subject of this paper.

The theory of singular perturbation and its applications is rich (Avrachenkov, 1999; Delebecque and Quadrat, 1981; Schweitzer, 1986; El-Azouzi and Altman, 2003). In particular, El-Azouzi and Altman (2003) studied a singular perturbation approach to approximating steady state solutions of a two-dimensional Markov chain. Specifically, a queueing analysis of packet dropping over a wireless link with retransmission was examined. The Markov chain consists of the channel state and the number of packets queued at the buffer. The idea is to investigate the effects of wireless channel conditions on the evolution of buffer sizes. Chydziński and Chróst (2011) presented analytical solutions for queue size distributions, loss ratio and throughput for Internet routers. Their analysis assumed a Poisson arrival process and the general type of the service time distribution (which is a more realistic model of packet processing time in an Internet router). However, the study does not consider energy consumption problems in multi-radio multi-channel network configurations.

On the other hand, the objective of this paper is to investigate the effects of queue pertubation arising from the evolution of energy consumption and buffer sizes, and the interference coupling on power control for multi-radio multi-channel wireless networks. This is motivated by the fact that energy availability for transmitting packets in queues do change generally at much different time-scales compared with that of buffer size evolution (Olwal, 2010). In fact, if the speed ratio between the buffer size and the energy level for transmission dynamics is represented by a sufficiently small positive parameter $\epsilon_{s}$, then the singular perturbation approach allows us to obtain steady state probabilities as a Taylor series of this parameter. On the other hand, weakly coupled theory and its application to large scale control systems can be found in the works of Shen and Gajic (1990), Sagara et al. (2008) or Mukaidani (2009). In these contributions, the emphasis is on modelling large scale dynamic control systems as differential Nash games (Gajic and Shen, 1993). In particular, assuming a large scale interconnected system, a strategy of one subsystem needs to be decoupled from strategies of others in the game so that the Nash equilibrium is attained (Mukaidani, 2009). In the same spirit, we model interchannel wireless interference as a weakly coupled control system and use the principle of Nash games to evaluate optimal transmission power levels by each user. To the best of our knowledge, the ideas presented in this paper have not been widely proposed in the literature for power control over multi-radio wireless backbone networks. The main contributions are (a) expression for the transmission probability and expected delay for the queue corresponding to each radio device, and (b) optimal control policies obtained as a Nash equilibrium of a game with radios at the same node as players. Table 1 depicts definitions of the abbreviations and notation often referred to in this paper.

The rest of this paper is organised as follows. In Section 2, we present singular perturbation, model analysis and the weakly coupled wireless system. In Section 3, the optimal power control problem is formulated. Section 4 summarises the proposed optimal power control algorithm. We provide numerical examples in Section 5 and conclude the paper in Section 6 with highlights of potential real applications of the proposed method. 
Table 1. Abbreviation and notation.

\begin{tabular}{|c|c|c|c|}
\hline Abbr. & Description & Notation & Description \\
\hline MAC & $\begin{array}{l}\text { medium } \\
\text { access control }\end{array}$ & $\epsilon_{s}$ & $\begin{array}{l}\text { singular per- } \\
\text { turbation (suf- } \\
\text { ficiently small } \\
\text { parameter) }\end{array}$ \\
\hline MRMC & $\begin{array}{l}\text { multiradio } \\
\text { multichannel }\end{array}$ & $\pi\left(\epsilon_{s}\right)$ & $\begin{array}{l}\text { Markov } \\
\text { chain steady } \\
\text { state prob. } \\
\text { distribution }\end{array}$ \\
\hline NIC & $\begin{array}{l}\text { network inter- } \\
\text { face card }\end{array}$ & $\psi\left(\epsilon_{s}\right)$ & $\begin{array}{l}\text { steady state } \\
\text { transmission } \\
\text { probability }\end{array}$ \\
\hline PMMUP & $\begin{array}{l}\text { power selec- } \\
\text { tion MRMC } \\
\text { unification } \\
\text { protocol }\end{array}$ & $\epsilon_{w}$ & $\begin{array}{l}\text { weakly } \\
\text { coupled suffi- } \\
\text { ciently small } \\
\text { parameter }\end{array}$ \\
\hline SPWC & $\begin{array}{l}\text { singularly } \\
\text { perturbed } \\
\text { weakly cou- } \\
\text { pled }\end{array}$ & $\tilde{\tilde{\epsilon}}$ & $\begin{array}{l}\text { singularly } \\
\text { perturbed } \\
\text { weakly } \\
\text { coupled (suf- } \\
\text { ficiently small } \\
\text { parameter) }\end{array}$ \\
\hline SWARRE & $\begin{array}{l}\text { SPWC Ricatti } \\
\text { equation }\end{array}$ & $\overline{\mathbf{P}_{\epsilon}}$ & $\begin{array}{l}\text { SWARRE sta- } \\
\text { bilising solu- } \\
\text { tion }\end{array}$ \\
\hline$\overline{\mathrm{UCG}}$ & $\begin{array}{l}\text { unified chan- } \\
\text { nel graph }\end{array}$ & $\overline{\mathbf{u}_{i}(t)}$ & $\begin{array}{l}\text { power control } \\
\text { sequence }\end{array}$ \\
\hline
\end{tabular}

\section{System model and analysis}

2.1. Queue system perturbation. In order to simplify our approach and still accomplish our study objectives, we describe a decentralized network model. Specifically, we assume that there are $N$ wireless links, each on a separate channel, emanating from one wireless MRMC node. This means that there are $N$ queues and $N$ times energy consumption for a multi-radio node. All transmitters of the same node share a common energy source and all packet transmissions are constrained by the total energy levels. For a sender-receiver node pair, the queues are depicted by Fig. 1. At the sender (respectively the receiver) packets from the PMMUP layer (respectively multiple queues) are striped (respectively resequenced) into multiple queues (respectively a PMMUP queue) (Olwal et al., 2009a). Moreover, each queue of an NIC utilizes the energy awareness and information from higher layers in order to perform an Admission Control Mechanism (ACM). The ACM selects the input rates to the finite-sized buffers before transmitting the packets over wireless channels.

Packets are considered to independently arrive at the multiple MAC and PHY queues and get transmitted after a finite duration of time depending on the buffer size, the available energy and the conditions of the wireless links. Therefore, during a given time-duration, the appli-

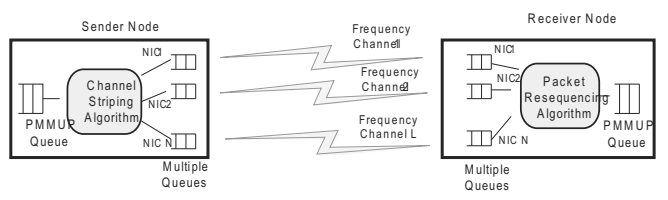

Fig. 1. Multiple queues of wireless multiple radio multiple channel systems.

cation generates packets according to a Bernoulli process (El-Azouzi and Altman, 2004), and such packets arrive at the queues with probability $\phi$. Each queue is assumed to have a finite size of $B$ packets. Suppose queues are initially nonempty, then new arriving packets are dropped when the queue is full, otherwise packets join the tail of the queue. Packets are then serviced and transmitted through the channel using an optimal transmission power sequence.

Set $i \in E$, where $E=\{1,2, \ldots, i, \ldots, E\}$, as the energy level available for transmitting a packet over a wireless medium by each transmit-receive NIC pair (user). Set $\varphi_{i}$, where $\varphi_{i} \in[0,1]$, as the probability of transmitting a packet with energy level $i$. The transition probability from energy state $X_{n}=i$ to another energy state $X_{n+1}=j$ during the time transition $[n, n+1]$ is given by

$$
\lambda_{i j}=\operatorname{Pr}\left(X_{n+1} \mid X_{n}=i\right),
$$

so that we can define

$$
\Lambda=\left[\begin{array}{cccc}
\lambda_{11} & \lambda_{12} & \cdots & \lambda_{1 E} \\
\lambda_{21} & \lambda_{22} & \cdots & \lambda_{2 E} \\
\ldots \ldots & \ldots & \ldots & \ldots \\
\lambda_{E 1} & \lambda_{E 2} & \cdots & \lambda_{E E}
\end{array}\right]
$$

as the transition matrix of the energy levels for transmission, where $\sum_{j=1}^{E} \lambda_{i j}=1$ with the probability distribution denoted by $\mathcal{V}=\left[\mathcal{V}_{1}, \mathcal{V}_{2}, \ldots, \mathcal{V}_{E}\right]$.

It should be noted that the power optimization phase requires the information about the queue and energy level dynamics in order to yield sequences of energy-efficient power levels. Such dynamics can be modelled as Markov chain processes described as follows.

Define $X(n)=\left\{X_{n}(i(n), j(n))\right\}$ as a twodimensional Markov chain sequence, where $i(n)$ and $j(n)$ are respectively the energy level available for packet transmission and the number of packets in the buffer at the $n$-th time step. Let the packet arrival and the energycharging process to each interface in step $n+1$ be independent of the chain $X(n)$. Arrivals are also assumed to occur at the end of each time step so that new arrivals cannot depart in the same time step that they arrive (Adisehu et al., 1996). Figure 2 shows a two-dimensional Markov chain evolution diagram with the transition probability matrix, $P_{T}(n)$, whose elements are $\lambda_{n, n+1}(i, j)$ for 


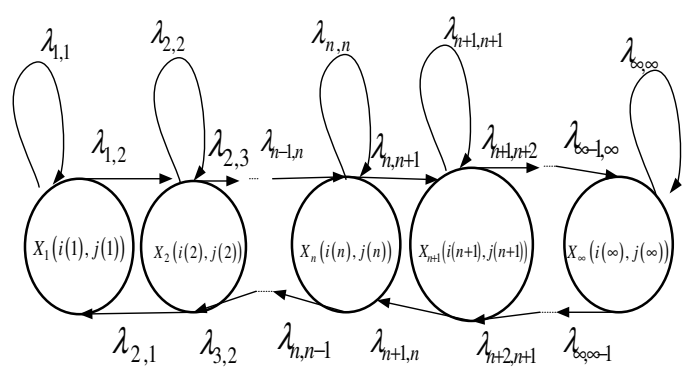

Fig. 2. Markov chain diagram.

all $i=1,2, \ldots, E$ and $j=0,1,2, \ldots, B$. The notation, $\lambda_{n, n+1}(i, j)$ represents the transition probability of the $i$-th energy level and the $j$-th buffer level from state at $n$ to state at $n+1$. We note that a similar Markov chain representation can be considered for other queues in a multi-queue system in Fig. 1. This is simply because all queues share common unified upper layers and energy source supply, but each transmission faces different wireless channel conditions, and hence a different energy consumption.

The transition probability $E(B+1) \times E(B+1)$ matrix of the Markov chain $X(n)$ is given by

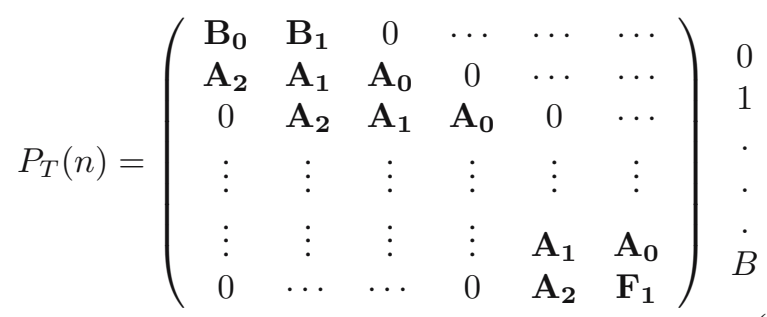

where $P_{T}(n)$ consists of $B+1$ block rows and $B+1$ block columns, each of size $E \times E$. The matrices $\mathbf{B}_{\mathbf{0}}, \mathbf{B}_{1}, \mathbf{A}_{\mathbf{0}}, \mathbf{A}_{\mathbf{1}}, \mathbf{A}_{\mathbf{2}}$ and $\mathbf{F}_{\mathbf{1}}$ are all $E \times E$ non-negative matrices given by

$$
\begin{aligned}
\mathbf{B}_{\mathbf{0}} & =\bar{\phi} \Lambda, \quad \mathbf{B}_{\mathbf{1}}=\phi \Lambda \\
\mathbf{A}_{\mathbf{0}} & =\operatorname{diag}\left(\phi \overline{\varphi_{i}}, i=1, \ldots, E\right) \Lambda, \\
\mathbf{A}_{\mathbf{1}} & =\operatorname{diag}\left(\phi \varphi_{i}+\bar{\phi} \overline{\varphi_{i}}, i=1, \ldots, E\right) \Lambda, \\
\mathbf{A}_{\mathbf{2}} & =\operatorname{diag}\left(\bar{\phi} \varphi_{i}, i=1, \ldots, E\right) \Lambda, \\
\mathbf{F}_{\mathbf{1}} & =\operatorname{diag}\left(\phi \varphi_{i}+\overline{\varphi_{i}}, i=1, \ldots, E\right) \Lambda .
\end{aligned}
$$

Here $\bar{\phi}=1-\phi$ and $\overline{\varphi_{i}}=1-\varphi_{i}$ denote respectively, the probability that no packet arrives in the queue and no packet is transmitted into the wireless channel when the available energy level is $i$. If we assume that the energy level transition matrix $\Lambda$ is irreducible and aperiodic and also that $\phi>0$, then the Markov chain $X(n)$ is aperiodic and has a single ergodic class (Schweitzer, 1986). We can then define a unique row vector of the steady state (or sta- tionary) probability distribution as

$$
\pi(i, j)=\lim _{n \rightarrow \infty} P_{T}(l(n)=i, b(n)=j),
$$

with $i=1,2, \cdots, E ; j=0,1, \ldots, B$ and $\pi(i, j) \in$ $\mathbb{R}^{1 \times i(j+1)}$. Let $\pi(i, j, \epsilon), i=1, \ldots, i, \ldots, E ; j=$ $1, \ldots, j, \ldots, B$, be the probability distribution of the state of the available energy and the number of packets in the system in the steady state. This probability distribution is uniquely determined by the following system:

$$
\begin{aligned}
\pi\left(\epsilon_{s}\right) P_{T}\left(\epsilon_{s}\right) & =\pi\left(\epsilon_{s}\right), \\
\pi\left(\epsilon_{s}\right) \mathbf{1} & =1, \\
\pi\left(\epsilon_{s}\right) & \geq 0,
\end{aligned}
$$

where $\epsilon_{s}$ denotes the singular perturbation factor depicting the speed ratio between energy and queue state evolutions. The singular perturbation factor is considered to differ from one radio to another since wireless channel conditions differ from one frequency channel to the other. The first order Taylor series approximation of the perturbed Markov chain transition matrix $X(n)$ defined in (2) can be represented as

$$
P_{T}\left(\epsilon_{s}\right)=Q_{0}+\epsilon_{s} Q_{1},
$$

where $Q_{0}$ is the probability of the transition matrix of the unperturbed Markov chain corresponding to the strong interactions of packet arrivals to and departures from the queues and $Q_{1}$ is the generator corresponding to the weak interactions, i.e.,

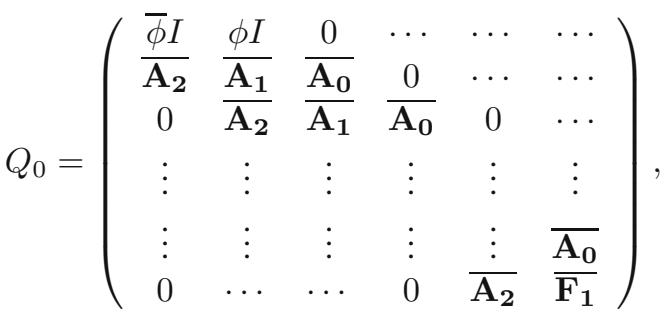

$$
\begin{aligned}
& Q_{1}=\left(\begin{array}{cccccc}
\widetilde{\mathbf{B}_{0}} & \widetilde{\mathbf{B}_{1}} & 0 & \cdots & \cdots & \cdots \\
\widetilde{\mathbf{A}_{2}} & \widetilde{\mathbf{A}_{1}} & \widetilde{\mathbf{A}_{0}} & 0 & \cdots & \ldots \\
0 & \widetilde{\mathbf{A}_{2}} & \widetilde{\mathbf{A}_{1}} & \widetilde{\mathbf{A}_{0}} & 0 & \ldots \\
\vdots & \vdots & \vdots & \vdots & \vdots & \vdots \\
\vdots & \vdots & \vdots & \vdots & \vdots & \widetilde{\mathbf{A}_{0}} \\
0 & \cdots & \cdots & 0 & \widetilde{\mathbf{A}_{2}} & \widetilde{\mathbf{F}_{1}}
\end{array}\right),
\end{aligned}
$$

where

$$
\begin{aligned}
& \overline{\mathbf{A}_{\mathbf{2}}}=\operatorname{diag}\left(\bar{\phi} \varphi_{i}, i=1, \ldots, E\right), \\
& \overline{\mathbf{A}_{1}}=\operatorname{diag}\left(\phi \varphi_{i}+\bar{\phi} \overline{\varphi_{i}}, i=1, \ldots, E\right), \\
& \overline{\mathbf{A}_{0}}=\operatorname{diag}\left(\phi \overline{\varphi_{i}}, i=1, \ldots, E\right), \\
& \overline{\mathbf{F}_{1}}=\operatorname{diag}\left(\phi \varphi_{i}+\overline{\varphi_{i}}, i=1, \ldots, E\right), \\
& \widetilde{\mathbf{B}_{0}}=\bar{\phi}\left(\Lambda_{1}\right),
\end{aligned}
$$




$$
\begin{aligned}
\widetilde{\mathbf{B}_{1}} & =\phi\left(\Lambda_{1}\right), \\
\widetilde{\mathbf{A}_{2}} & =\operatorname{diag}\left(\bar{\phi} \varphi_{i}, i=1, \ldots, E\right) \Lambda_{1}, \\
\widetilde{\mathbf{A}_{1}} & =\operatorname{diag}\left(\phi \varphi_{i} \bar{\phi} \overline{\varphi_{i}}, i=1, \ldots, E\right) \Lambda_{1}, \\
\widetilde{\mathbf{A}_{0}} & =\phi \operatorname{diag}\left(\phi \overline{\varphi_{i}}, i=1, \ldots, E\right) \Lambda_{1}, \\
\widetilde{\mathbf{F}_{1}} & =\operatorname{diag}\left(\phi \varphi_{i}+\overline{\varphi_{i}}, i=1, \ldots, E\right) \Lambda_{1},
\end{aligned}
$$

Here,

$$
\Lambda\left(\epsilon_{s}\right)=I+\epsilon_{s} \Lambda_{1},
$$

where $\Lambda_{1}$ is the generator matrix (Avrachenkov, 1999) representing an aggregated Markov chain $X(n)$.

The model in (2) to (7) gives the perturbation problem under the assumption that there exists an ergodic class (i.e., the one which has exactly one closed communicating set of states), and $Q_{0}$ contains $E$ sub-chains ( $E$ ergodic class).

The stationary probability $\pi\left(i, j, \epsilon_{s}\right)$ of the perturbed Markov chain from (3) and (4) has a Taylor series expansion,

$$
\pi\left(i, j, \epsilon_{s}\right)=\sum_{n=0}^{\infty} \pi^{(n)}(i, j) \epsilon_{s}^{n},
$$

where $\epsilon_{s}$ is the $n$-th order singularly-perturbed parameter. From (1), the aggregate Markov chain probability distribution was denoted by $\overline{\mathcal{V}}=\left[\overline{\mathcal{V}}_{1}, \overline{\mathcal{V}}_{2}, \ldots, \overline{\mathcal{V}}_{E}\right]$. The unperturbed stationary probability is then given by

$$
\pi^{(0)}(i, j)=\overline{\mathcal{V}}_{i} \nu_{\zeta_{i}}(j),
$$

where $\nu_{\zeta_{i}}$ is the probability distribution of the recurrent class $\zeta_{i}$, i.e., $\sum_{j=0}^{B} \zeta_{i}(j)=1$ (El-Azouzi and Altman, 2004).

2.2. Packet transmission and delay analysis. The following analysis illustrates the probability of packet transmission and the expected delay at each queue. Their impacts on transmission energy consumption at each multi-radio node are also shown. The motivation is to have a power control solution which is both energy source and queue delay aware for the purpose of implementing an energy efficient network.

Lemma 1. For each $i=1,2, \ldots, E$, the stationary distribution $\nu_{\zeta_{i}}(j)$ of the recurrent class $\zeta_{i}$ is given as follows

1. If $i \notin E_{H} \cup E_{L}$, then

$\nu_{\zeta_{i}}(j+1)=\frac{\phi^{j+1}\left(1-\varphi_{i}\right)^{j}}{\varphi_{i}^{j+1}(1-\phi)^{j+1}} \nu_{\zeta_{i}}(0), \quad j=1,2, \ldots, B$.

Here

$$
\nu_{\zeta_{i}}(0)=\frac{\varphi_{i}-\phi}{\varphi_{i}-\phi \alpha^{B}}
$$

with

$$
\alpha=\frac{\phi\left(1-\varphi_{i}\right)}{\varphi_{i}(1-\phi)} .
$$

$E_{H}$ and $E_{L}$ are respectively sets of the energy levels for the packet transmission higher and lower than the minimum required for the successful packet transmission.

2. If $i \in E_{H}$, then $\nu_{\zeta_{i}}(0)=1-\phi$ and $\nu_{\zeta_{i}}(1)=\phi$.

3. If $i \in E_{L}$, then $\nu_{\zeta_{i}}(B)=1$.

Proof. If the level of the available energy for the packet transmission is $i \notin E_{H} \cup E_{L}$, then we first consider $\nu_{\zeta_{i}}(1)$ in terms of $\nu_{\zeta_{i}}(0)$, i.e.,

$$
\nu_{\zeta_{i}}(1)=\frac{1}{1-\varphi_{i}} \alpha \nu_{\zeta_{i}}(0) .
$$

Consequently by induction one gets

$$
\begin{gathered}
\nu_{\zeta_{i}}(j)=\frac{1}{1-\varphi_{i}} \alpha^{j} \nu_{\zeta_{i}}(0), \quad j=1, \ldots, B, \\
\alpha=\frac{\phi\left(1-\varphi_{i}\right)}{\varphi_{i}(1-\phi)} .
\end{gathered}
$$

Thus,

$$
\nu_{\zeta_{i}}(j+1)=\frac{1}{1-\varphi_{i}} \alpha^{j+1} \nu_{\zeta_{i}}(0) .
$$

Alternatively,

$$
\nu_{\zeta_{i}}(j+1)=\frac{\phi^{j+1}\left(1-\varphi_{i}\right)^{j}}{\varphi_{i}^{j+1}(1-\phi)^{j+1}} \nu_{\zeta_{i}}(0), j=1, \cdots, B .
$$

Using the normalization condition

$$
\sum_{j=0}^{B} \zeta_{i}(j)=1,
$$

we can obtain $\nu_{\zeta_{i}}(0)$ by summing over all $j=$ $0,1,2, \ldots, B$,

$$
\nu_{\zeta_{i}}(0)=\frac{1}{1+\frac{1}{1-\varphi_{i}} \alpha \frac{1-\alpha B}{1-\alpha}}=\frac{\varphi_{i}-\phi}{\varphi_{i}-\phi \alpha^{B}} .
$$

Suppose the level of the available energy is $i \in E_{H}$, given that there is no packet in the queue. Then the condition $\nu_{\zeta_{i}}(0)=1-\phi=\bar{\phi}$ holds. Consequently, one packet arrives with the probability $\phi$, implying that $\nu_{\zeta_{i}}(1)=\phi$. In a similar treatment, if the energy level is $i \in E_{L}$, given a full buffer then the stationary distribution of the recurrent class $\zeta_{i}$ is given by $\nu_{\zeta_{i}}(B)=1$.

Lemma 2. Assuming that there exists a perturbed Markov chain $X_{n}\left(i, j, \epsilon_{s}\right)$, the perturbed steady state transmission probability is given as follows:

$$
\begin{aligned}
\Psi\left(\epsilon_{s}\right)= & \sum_{i, j} \frac{\phi^{j+1}\left(1-\varphi_{i}\right)^{j-1} \varphi_{i}^{B+1}(1-\phi)^{B}\left(\varphi_{i}-\phi\right)}{\varphi_{i}^{B+j}(1-\phi)^{B+j}-\phi^{B+1}\left(1-\varphi_{i}\right)^{B-1}} \\
& +\phi \sum_{i, j} \sum_{n=1}^{\infty} \pi^{(n)}(i, j) \epsilon_{s}^{n} \varphi_{i},
\end{aligned}
$$


where

$$
\begin{aligned}
\pi^{(n)}= & \mathcal{V}\left[\mathbf{R}\left(\mathbf{Q}_{1} \Delta_{0}\right)^{n}\right. \\
& \left.+\sum_{k=1}^{n-1}\left(\mathbf{R} \mathbf{Q}_{1} \Delta_{0} \tilde{\mathbf{R}} \Delta_{1}\right)^{k}\left(\mathbf{Q}_{1} \Delta_{0}\right)^{n-k}\right]
\end{aligned}
$$

Here, $\mathbf{R} \in \mathbb{R}^{(E+1) \times(E+1)(B+1)}$ denotes a matrix whose rows form a stationary distribution of the Markov chain $\mathbf{Q}_{0}$, i.e., $\mathbf{R}=\operatorname{diag}\left(\nu_{\zeta_{i}}, i=0,1, \ldots, E\right) . \quad \tilde{\mathbf{R}} \in$ $\mathbb{R}^{(E+1)(B+1) \times(E+1)}$ denotes a matrix of eigenvectors corresponding to the zero eigenvalue of the unperturbed generator $\mathbf{Q}_{0}-I$, i.e., $\tilde{\mathbf{R}}=\left(\theta_{\zeta_{0}}, \theta_{\zeta_{1}}, \ldots, \theta_{\zeta_{E}}\right) . \Delta_{0}$ denotes $a$ deviation matrix of the unperturbed Markov chain $\Delta_{0}=$ $\left[I-\mathbf{Q}_{0}+\tilde{\mathbf{R}} \mathbf{R}\right]^{-1}-\tilde{\mathbf{R}} \mathbf{R} . \Delta_{1}=\left[-\Lambda_{1}+\Lambda^{*}\right]^{-1}-\Lambda^{*} d e-$ notes a deviation of the aggregated Markov chain, where $\Lambda^{*}=\mu_{E} \mathcal{V}$, with $\mu_{E}=(1, \ldots, 1)^{T} \in \mathbb{R}^{E+1}($ El-Azouzi and Altman, 2003).

Proof. By definition and Lemma 1, the steady state transmission probability $\Psi\left(\epsilon_{s}\right)$ is given by

$$
\begin{aligned}
\Psi\left(\epsilon_{s}\right)= & \phi \sum_{(i, j)} \sum_{n=0}^{\infty} \pi^{(n)}(i, j) \epsilon_{s}^{n} \varphi_{i} \\
& (i=1,2, \ldots, E, j=0,1,2, \ldots, B \\
= & \phi \sum_{(i, j)} \pi^{(0)}(i, j) \epsilon_{s}^{0} \varphi_{i}+\phi \sum_{(i, j)} \sum_{n=1}^{\infty} \pi^{(n)}(i, j) \epsilon_{s}^{n} \varphi_{i} \\
= & \phi \sum_{(i, j)} \mathcal{V}_{i} \nu_{\zeta_{i}}(j) \varphi_{i}+\phi \sum_{(i, j)} \sum_{n=1}^{\infty} \pi^{(n)}(i, j) \epsilon_{s}^{n} \varphi_{i} \\
= & \phi \sum_{(i, j)} \mathcal{V}_{i} \frac{\alpha^{j} \nu_{\zeta_{i}}(0)}{1-\varphi_{i}} \varphi_{i}+\phi \sum_{(i, j)} \sum_{n=1}^{\infty} \pi^{(n)}(i, j) \epsilon_{s}^{n} \varphi_{i} \\
= & \sum_{(i, j)} \mathcal{V}_{i} \frac{\phi^{j+1}\left(1-\varphi_{i}\right)^{j-1} \varphi_{i}^{B+1}(1-\phi)^{B}\left(\varphi_{i}-\phi\right)}{\varphi_{i}^{B+j}(1-\phi)^{B+j}-\phi^{B+1}\left(1-\varphi_{i}\right)^{B-1}} \\
& +\phi \sum_{(i, j)} \sum_{n=1}^{\infty} \pi^{(n)}(i, j) \epsilon_{s}^{n} \varphi_{i}
\end{aligned}
$$

with $\pi^{(n)}$ being recursively evaluated as given in Lemma 2.

Lemma 3. Assuming that there exists a perturbed Markov chain $X_{n}\left(i, j, \epsilon_{s}\right)$, the expected delay in a singularly perturbed queue, $E_{D}\left(\epsilon_{s}\right)$, is given as follows:

$$
E_{D}\left(\epsilon_{s}\right)=\frac{N_{u}}{D_{e}}
$$

where

$$
\begin{aligned}
N_{u}= & \sum_{(i, j)} \mathcal{V}_{i} \frac{\phi^{j}\left(1-\varphi_{i}\right)^{j-1} \varphi_{i}^{B}(1-\phi)^{B}\left(\varphi_{i}-\phi\right) j}{\varphi_{i}^{B+j}(1-\phi)^{B+j}-\phi^{B+1}\left(1-\varphi_{i}\right)^{B-1}} \\
& +\sum_{(i, j)} \sum_{n=1}^{\infty} \pi^{(n)}(i, j) \epsilon_{s}^{n} j \\
& i=1,2, \ldots, E, j=0,1,2, \ldots, B . \\
D_{e}= & \phi-\sum_{i \notin E_{H} \cup E_{L}} \mathcal{V}_{i} \frac{\phi^{B+1}\left(1-\varphi_{i}\right)^{B}}{\left(\varphi_{i}(1-\phi)\right)^{B}}+\sum_{i \in E_{L}} \mathcal{V}_{i} \\
& +\sum_{i=1} \sum_{n=1}^{\infty} \pi^{(n)}(i, B) \epsilon_{s}^{n},
\end{aligned}
$$

with $\pi^{(n)}$ given by results of Lemma 2.

Proof. By definition, the average number of packets in the queue is given by

$$
\begin{aligned}
N_{u}= & E\left[X_{n}\right]=\sum_{(i, j)} \pi\left(i, j, \epsilon_{s}\right) j \\
= & \sum_{(i, j)} \sum_{n=0}^{\infty} \pi^{(n)}(i, j) \epsilon_{s}^{n} j \\
= & \sum_{(i, j)} \pi^{(0)}(i, j) j+\sum_{(i, j)} \sum_{n=1}^{\infty} \pi^{(n)}(i, j) \epsilon_{s}^{n} j, \\
& i=1,2, \ldots, E, \quad j=0,1,2, \ldots, B .
\end{aligned}
$$

Using results from Lemma 1, one proceeds to the expression of $N_{u}$ given in Lemma 3.

Let $\phi^{*}$ be the packet acceptance rate into the queue at steady state. Define $\phi^{*}$ as

$$
\begin{aligned}
\phi^{*}= & D_{e}=\phi\left(1-\sum_{i=1}^{E} \pi\left(i, B, \epsilon_{s}\right) \varphi_{i}\right) \\
= & \phi\left(1-\sum_{i=1}^{E} \sum_{n=0}^{\infty} \pi^{(n)}(i, B) \epsilon_{s}^{n} \varphi_{i}\right) \\
= & \phi\left(1-\sum_{i=1}^{E} \pi^{(0)}(i, B) \varphi_{i}\right. \\
& \left.-\sum_{i=1}^{E} \sum_{n=1}^{\infty} \pi^{(n)}(i, B) \epsilon_{s}^{n} \varphi_{i}\right) .
\end{aligned}
$$

Applying results of Lemma 1, we obtain the expression of $D_{e}$ given in Lemma 3.

Using Little's theorem (Avrachenkov, 1999), the average delay in a perturbed queue is given by

$$
E_{D}\left(\epsilon_{s}\right)=\frac{N_{u}}{D_{e}}
$$

It should be noted from results of Lemmas 1 and 2 that the steady state transmission probability of a packet from 
the queue and the expected packet queue delay are decomposable into an unperturbed component (i.e., independent of $\epsilon_{s}$ ) and a perturbed component expressed in terms of a higher-order Taylor series expansion (i.e., dependent on $\left.\epsilon_{s}\right)$. A higher-order Taylor series expansion with a sufficiently large step size $n$ results in significantly small values of perturbation hence such terms can be neglected. The first-order Taylor series expansion in terms of the perturbation, factor presents a reliable approximation of the exact steady state probability distribution of the Markov chain $X_{s}\left(i, j, \epsilon_{s}\right)$.

2.3. Weakly coupled wireless system. In theory, simultaneously transmitting links on different nonoverlapping channels are expected not to conflict with each other. However, links emanating from the same node of a multi-radio wireless system do conflict with each other due to the close separation vicinity. The colocated wireless multi-radio multi-channel networks do have negligible interference conflicts but show significant coupling effects due to the radiated power generated by sending nodes. The radiated power coupling across multiple channels results in a loss in signal strength due to interchannel interference and hence packet losses over multichannel wireless links. Packet losses lead to packet retransmissions, and hence packet propagation delays along a link(s). Retransmissions, in turn, lead to a high energy consumption. In order to take into account the problems of retransmissions, one can model the cross-channel wireless interference (interaction) as a weakly coupled system (Gajic and Shen, 1993). Each NIC operating on a particular channel can then select a transmission power level that depends on a sufficiently small positive parameter denoted as $\epsilon_{w}$.

In particular, consider a two-dimensional node placement consisting of two collocated wireless channels labelled $i$ and $j$. The channels allow simultaneous radial transmissions as depicted by Fig. 3. The figure illustrates two simultaneously collocated transmitting users $i$ and $j$ described by the pair $T X R_{i}-R X R_{i}$ of infinitesimally small radiating points and the pair $T X R_{j}-R X R_{j}$, respectively. Specifically, transmitters $i$ and $j$ are assumed to radiate signals omni-directionally with radii $d_{i}$ and $d_{j}$ and cause strong inter-channel connections due to their close vicinity. Here, $d_{i}$ refers to the conflict edge from a sender on channel $i$ to a receiver on channel $i$, and $d_{j}$ is the conflict edge from a sender on channel $j$ to a receiver on channel $i$. The region denoted by $A_{\epsilon}$ is the weakly coupling region described by $d_{i}$ and $d_{j}$ as well as the coupling beamwidth angles $\theta_{i}$ and $\theta_{j}$ in radians.

It should be noted that, since a power coupling is considered, the weak coupling factor can be derived as a function of the region or surface $A_{\epsilon}$, i.e., $O\left(d_{i j}^{2}\right)$, where $d_{i j}$ is the distance between points $i$ and $j$.

From the geometrical analysis of Fig. 3, it is easy to

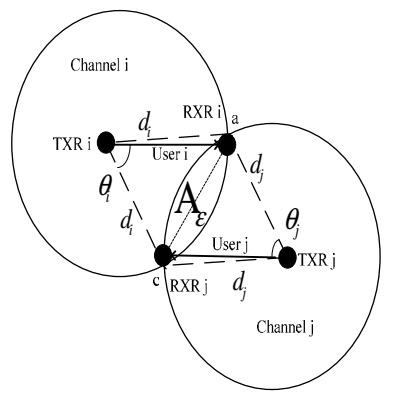

Fig. 3. Two realistic geographically neighbouring channels with two independent users from the same node.

show that the weak coupling parameter is obtained as

$$
\epsilon_{i j}=\frac{A_{\epsilon i}}{A_{\epsilon}}=\frac{d_{i}^{2}\left[\theta_{i}-\frac{\sin \theta_{i}}{\sqrt{2}}\right]}{d_{i}^{2}\left[\theta_{i}-\frac{\sin \theta_{i}}{\sqrt{2}}\right]+d_{j}^{2}\left[\theta_{j}-\frac{\sin \theta_{j}}{\sqrt{2}}\right]}
$$

and

$$
\epsilon_{j i}=\frac{A_{\epsilon j}}{A_{\epsilon}}=\frac{d_{j}^{2}\left[\theta_{j}-\frac{\sin \theta_{j}}{\sqrt{2}}\right]}{d_{i}^{2}\left[\theta_{i}-\frac{\sin \theta_{i}}{\sqrt{2}}\right]+d_{j}^{2}\left[\theta_{j}-\frac{\sin \theta_{j}}{\sqrt{2}}\right]}
$$

Thus, the weakly coupled scalar is generally a function of the square of the transmission radius and the coupling-sector angle. That is, the longer the transmission radius (i.e., range) and the wider the beamwidth (i.e., coupling angle), the stronger the interference coupling and the higher the transmission power consumption. Consequently, the weak coupling parameter can then be bounded by $0<\epsilon_{i j}=\epsilon_{w}<1$. On the other hand, the sectored angle has a bound, $0 \leq \theta \leq 2 \pi$.

In a wireless MRMC power control system one seeks to find the impact of weakly coupled systems with slow and fast variables on the optimal selection of the transmission power levels. The obtained optimal power control solutions are given in terms of a ratio of two small positive parameters. That is, the notation $0<\epsilon=\epsilon_{w} / \epsilon_{s}<\infty$ signifies that the MRMC system is both weakly coupled and singularly perturbed. If each transmit-receive NIC pair seeks to obtain a transmission power control signal $u_{l}^{*}(t)$ that drives multiple states of the system to steady states, then the dynamic power control law for the $l$-th channel is (Olwal et al., 2009b; 2009c)

$$
\begin{aligned}
& p_{l}(t+1) \\
& = \begin{cases}p_{l}(t)+f_{l}(\mathbf{x}) & \forall \mathbf{x} \text { and } 0<\epsilon=\epsilon_{w} / \epsilon_{s}<\infty, \\
0 & \text { otherwise, }\end{cases}
\end{aligned}
$$

where $f_{l}(\mathbf{x})=f_{l}\left(\beta_{l}(t), I_{l}(t), \Gamma_{l}(t)\right)$ denotes a nonlinear function representing the $l$-th channel with multiple link 
states. Here, $\beta_{l}(t), I_{l}(t), \Gamma_{l}(t)$ denote respectively the actual SINR, the aggregate co-channel network interference, the link packet rate and the connectivity range during the time slot $t$

It should be noted from (15) that the dynamic power control in wireless MRMC systems is constrained by several complexities such as the high dimension of these radio-channel pairs and the high order of the state variables describing the communication performance measures. Concerning the problem of dimension, the study can be simplified by decomposing the system into separate but interconnected single channels (i.e., each being a set of subsystems), also known as the Unified Channel Graphs (UCGs) (Olwal et al., 2009c). Modelling the networks into UCGs provides the advantage of utilising decentralization techniques (i.e., computationally simple) in dealing with large-scale systems (Gajic and Shen, 1993). Consequently, the higher-order state variables are then reflected by a wireless interconnection coupling parameter model (Shen and Gajic, 1990). This motivates us to exploit such a procedure so as to re-formulate the classical transmission power control problem for the disjoint UCGs in terms of Nash equilibrium differential games.

The decision to use game theoretical approaches is triggered by the fact that, within each UCG, each user/player desires to minimize interference it causes to the network, reduce the energy consumption and improve the network scalability. Also, achieving similar objectives is necessary among different UCGs experiencing vast wireless channel conditions. Thus, it is imperative for users to form a coalition of behaviours that ultimately result in a Nash equilibrium so that optimal solutions be attained. Although numerous works exist in the literature that use game theoretical approaches to optimal control problems (Olwal, 2010), not much research is known that combines techniques of Singular Perturbation and Weak Coupling (SPWC) of multi-channel multi-radio networks. This research gap is the foundation of this contribution.

\section{Problem formulation}

Consider the SPWC wireless MRMC mesh node with sender-receiver pairs (subsystems). The decentralised linear dynamic control system has the form consistent with both weakly coupled and singularly perturbed systems. The large-scale linear dynamic system is then written as (Shen and Gajic, 1990; Sagara et al., 2008; Mukaidani, 2009)

$$
\begin{aligned}
& \mathbf{x}_{i}(t+1) \\
& =\mathbf{A}_{i i}(\epsilon) \mathbf{x}_{i}(t)+\mathbf{B}_{i i}(\epsilon) \mathbf{u}_{i}(t)+\mathbf{W}_{i i}(\epsilon) \mathbf{w}_{i}(t) \\
& \quad+\sum_{j=1, j \neq i}^{N} \epsilon_{i j} \mathbf{A}_{i j} \mathbf{x}_{j}(t)+\sum_{j=1, j \neq i}^{N} \epsilon_{i j} \mathbf{B}_{i j} \mathbf{u}_{j}(t)
\end{aligned}
$$

$$
\begin{gathered}
+\sum_{j=1, j \neq i}^{N} \epsilon_{i j} \mathbf{W}_{i j} \mathbf{w}_{j}(t), \\
\mathbf{x}_{i}(0)=\mathbf{x}_{i}^{0}, \quad i=1, \ldots, N, \\
\mathbf{y}_{i}(t)=\mathbf{C}_{i i}(\epsilon) \mathbf{x}_{i}(t) \sum_{j=1, j \neq i}^{N} \epsilon_{i j} \mathbf{C}_{i j} \mathbf{x}_{j}(t)+\mathbf{v}_{i}(t),
\end{gathered}
$$

where $\mathbf{x}_{i} \in \mathbb{R}^{n_{i}}$ represents the state vector of the $i$-th MRMC subsystem, $\mathbf{u}_{i} \in \mathbb{R}^{m_{i}}$ is the control input (that represents the transmission power sequence constrained by the maximum power output of the radio device) of the $i$-th MRMC subsystem, $\mathbf{w}_{i} \in \mathbb{R}^{q_{i}}$ represents the zero mean disturbance noise vector to the $i$-th MRMC subsystem, $\mathbf{y}_{i} \in \mathbb{R}^{j_{i}}$ represents the observed output and $\mathbf{v}_{i} \in \mathbb{R}^{l_{i}}$ is the zero mean measurement noise vector. The white noise processes $\mathbf{w}_{i} \in \mathbb{R}^{q_{i}}$ and $\mathbf{v}_{i} \in \mathbb{R}^{l_{i}}$ are independent and mutually uncorrelated with intensities $\Theta_{w}>0$ and $\Theta_{v}>0$, respectively. The coefficient matrices of Eqn. (16) are evaluated according to our previous work (Olwal et al., 2009c).

Suppose the partitioned matrices for a wireless MRMC communication system with $0<\epsilon=\epsilon_{w} / \epsilon_{s}<\infty$ be defined according to the structure

$$
\begin{aligned}
& \mathbf{A}_{\epsilon}=\left[\begin{array}{cccc}
\mathbf{A}_{11}(\epsilon) & \epsilon_{12} \mathbf{A}_{12} & \cdots & \epsilon_{1 N} \mathbf{A}_{1 N} \\
\epsilon_{21} \mathbf{A}_{21} & \mathbf{A}_{22}(\epsilon) & \cdots & \epsilon_{2 N} \mathbf{A}_{2 N} \\
\ldots \ldots \ldots \ldots \ldots \ldots \ldots \ldots & \ldots \ldots \ldots \ldots \ldots \\
\epsilon_{N 1} \mathbf{A}_{N 1} & \epsilon_{N 2} \mathbf{A}_{N 2} & \cdots & \mathbf{A}_{N N}(\epsilon)
\end{array}\right], \\
& \mathbf{B}_{i \epsilon}=\left[\begin{array}{l}
\epsilon^{1-\delta_{1 i}} \mathbf{B}_{1 i} \\
\epsilon^{1-\delta_{2 i}} \mathbf{B}_{2 i} \\
\cdot \\
\epsilon^{1-\delta_{N i}} \mathbf{B}_{N i}
\end{array}\right] \\
& \delta_{i j}=\left\{\begin{array}{lll}
0 & \text { if } \quad i \neq j \\
1 & \text { if } \quad i=j
\end{array}\right.
\end{aligned}
$$

$$
\begin{aligned}
& \mathbf{W}_{\epsilon}=\left[\begin{array}{cccc}
\mathbf{W}_{11}(\epsilon) & \epsilon_{12} \mathbf{W}_{12} & \cdots & \epsilon_{1 N} \mathbf{W}_{1 N} \\
\epsilon_{21} \mathbf{W}_{21} & \mathbf{W}_{22}(\epsilon) & \cdots & \epsilon_{2 N} \mathbf{W}_{2 N} \\
\ldots \ldots \ldots \ldots \ldots \ldots \ldots \ldots \ldots \ldots \ldots \ldots \ldots \ldots & \ldots \ldots
\end{array}\right] \\
& \mathbf{C}_{\epsilon}=\left[\begin{array}{cccc}
\mathbf{C}_{11}(\epsilon) & \epsilon_{12} \mathbf{C}_{12} & \cdots & \epsilon_{1 N} \mathbf{C}_{1 N} \\
\epsilon_{21} \mathbf{C}_{21} & \mathbf{C}_{22}(\epsilon) & \cdots & \epsilon_{2 N} \mathbf{C}_{2 N} \\
\ldots \ldots \ldots \ldots \ldots \ldots \ldots \ldots \ldots \ldots \ldots \ldots \ldots & \ldots \ldots \ldots
\end{array}\right]
\end{aligned}
$$

From the structures, it can be discerned that each strategic user is faced with the minimization problem 
along the trajectories of a linear dynamic system in (16),

$$
\begin{aligned}
& J_{i}\left(u_{1}, \ldots, u_{N}, \mathbf{w}, \mathbf{x}(0)\right) \\
& =\frac{1}{2} E\left\{\operatorname { l i m } _ { t \rightarrow \infty } \frac { 1 } { t } \sum _ { \tau = 0 } ^ { t - 1 } \left[\mathbf{z}^{T}(\tau) \mathbf{z}(\tau)+\mathbf{u}_{i}^{T}(\tau) \mathbf{R}_{i i} \mathbf{u}_{i}(\tau)\right.\right. \\
& \left.\left.\quad+\sum_{j=1, j \neq i}^{N} \epsilon_{i j} \mathbf{u}_{j}^{T}(\tau) \mathbf{R}_{i j} \mathbf{u}_{j}(\tau)-\mathbf{w}^{t}(t) \Theta_{w i \epsilon} \mathbf{w}(t)\right]\right\},
\end{aligned}
$$

where $\mathbf{z} \in \mathbb{R}^{s}$ is the controlled output of the dimension $s$, given by (Gajic and Shen, 1993)

$$
\mathbf{z}_{i}(t)=\mathbf{D}_{i i}(\epsilon)+\sum_{j=1, j \neq i}^{N} \epsilon_{i j} \mathbf{D}_{i j} \mathbf{x}_{j}(t)
$$

with

$$
\mathbf{D}_{\epsilon}=\left[\begin{array}{cccc}
\mathbf{D}_{11}(\epsilon) & \epsilon_{12} \mathbf{D}_{12} & \cdots & \epsilon_{1 N} \mathbf{D}_{1 N} \\
\epsilon_{21} \mathbf{D}_{21} & \mathbf{D}_{22}(\epsilon) & \cdots & \epsilon_{2 N} \mathbf{D}_{2 N} \\
\ldots \ldots \ldots & \ldots \ldots \ldots \ldots \ldots & \ldots \ldots \ldots \\
\cdots \ldots & \ldots \ldots & \ldots \\
\epsilon_{N 1} \mathbf{D}_{N 1} & \epsilon_{N 2} \mathbf{D}_{N 2} & \cdots & \mathbf{D}_{N N}(\epsilon)
\end{array}\right]
$$

$\mathbf{R}_{i i}=\mathbf{R}_{i i}^{T} \in \mathbb{R}^{m_{i} \times m_{i}}$ being a symmetric positive definite matrix, $\mathbf{R}_{i j}=\mathbf{R}_{i j}^{T} \geq 0 \in \mathbb{R}^{m_{j} \times m_{j}}$, symmetric positive semi-definite matrices, $\Theta_{w i \epsilon}=$ block diag $\left(\epsilon_{i 1}^{-\left(1-\delta_{i 1}\right)} \Theta_{w i 1} \ldots \epsilon_{i N}^{-\left(1-\delta_{i N}\right)} \Theta_{w i N}\right) \geq 0 \in$ $\mathbb{R}^{\bar{q} \times \bar{q}}, i, j=1, \cdots, N$, representing the logical users of the multi-radio multi-channel system.

3.1. Nash strategies. The optimal solution to the given problem (18) with the conflict of interest and simultaneous decision making leads to the so-called Nash strategies (Gajic and Shen, 1993) $\mathbf{u}_{1}^{*}, \ldots, \mathbf{u}_{i}^{*}, \ldots, \mathbf{u}_{N}^{*}$ satisfying

$$
\begin{aligned}
J_{i}\left(\mathbf{u}_{1}^{*}, \ldots, \mathbf{u}_{i}^{*}, \ldots\right. & \left., \mathbf{u}_{N}^{*}, \mathbf{x}(0)\right) \\
& \leq \forall i J_{i}\left(\mathbf{u}_{1}^{*}, \cdots, \mathbf{u}_{i}, \cdots, \mathbf{u}_{N}^{*} \mathbf{x}(0)\right),
\end{aligned}
$$

Assumption 1. Each user has optimal closed-loop Nash strategies given by

$$
\mathbf{u}_{i}^{*}=-\mathbf{F}_{i \epsilon}^{*} \mathbf{x}(t) .
$$

Here, $\mathbf{F}_{i \epsilon}^{*}$ is the regulator feedback gain defined as

$$
\mathbf{F}_{i \epsilon}^{*}=\left[\epsilon^{1-\delta_{1 i}} \mathbf{F}_{1 i} \epsilon^{1-\delta_{2 i}} \mathbf{F}_{2 i} \cdots \epsilon^{1-\delta_{N i}} \mathbf{F}_{N i}\right] \in \mathbb{R}^{\bar{n}},
$$

with $\bar{n}=\sum_{i=1}^{N} n_{i}, n_{i}$ being the size of the vector $x_{i}$. by

Define the $N$-tuple discrete in time Nash strategies

$$
\begin{array}{r}
\mathbf{u}_{i}^{*}(t)=-\mathbf{F}_{i \epsilon}^{*} \mathbf{x}(t) \\
=-\left(\mathbf{R}_{i i}+\mathbf{B}_{i \epsilon}^{T} \mathbf{P}_{i \epsilon} \mathbf{B}_{i \epsilon}\right)^{-1} \mathbf{B}_{i \epsilon}^{T} \mathbf{P}_{i \epsilon} \mathbf{A}_{\epsilon} \mathbf{x}(t), \\
i=1, \ldots, N
\end{array}
$$

where $\left(\mathbf{F}_{1 \epsilon}^{*}, \ldots, \mathbf{F}_{N \epsilon}^{*}\right) \in \mathbf{F}_{N}$ and $N$-tuple $\mathbf{u}_{i}^{*}(t)$ form a soft constrained Nash equilibrium represented as

$$
J_{i}\left(\mathbf{F}_{1 \epsilon}^{*} \mathbf{x}, \ldots, \mathbf{F}_{N \epsilon}^{*} \mathbf{x}, \mathbf{x}(0)\right)=\mathbf{x}(0)^{T} \mathbf{P}_{i \epsilon} \mathbf{x}(0) .
$$

Here, $\mathbf{P}_{i \epsilon}$ is a positive semi-definite stabilizing solution of the Discrete-time Algebraic Regulator Riccati equation (DARRE) with the following structure:

$$
\begin{aligned}
\mathbf{P}_{i \epsilon} & =\mathbf{P}_{i \epsilon}^{T} \\
& =\left[\begin{array}{cccc}
\epsilon_{i 1}^{1-\delta_{i 1}} \mathbf{P}_{i 1} & \epsilon_{i 2} \mathbf{P}_{i 12} & \ldots & \epsilon_{i N} \mathbf{P}_{i 1 N} \\
\epsilon_{i 2} \mathbf{P}_{i 12}^{T} & \epsilon_{i 2}^{1-\delta_{i 2}} \mathbf{P}_{i 2} & \ldots & \epsilon_{i N} \mathbf{P}_{i 2 N} \\
\ldots & \ldots & \ldots & \ldots \\
\epsilon_{i N} \mathbf{P}_{i 1 N}^{T} & \epsilon_{i N} \mathbf{P}_{i 2 N}^{T} & \ldots & \epsilon^{1-\delta_{i N}} \mathbf{P}_{i N}
\end{array}\right]
\end{aligned}
$$

where the DARRE is given by

$$
\begin{aligned}
\mathbf{P}_{\epsilon}= & \mathbf{D}_{\epsilon}^{T} \mathbf{D}_{\epsilon}+\mathbf{A}_{\epsilon}^{T} \mathbf{P}_{\epsilon} \mathbf{A}_{\epsilon} \\
& -\mathbf{A}_{\epsilon}^{T} \mathbf{P}_{\epsilon} \mathbf{B}_{\epsilon}\left(\mathbf{R}_{\epsilon}+\mathbf{B}_{\epsilon}^{T} \mathbf{P}_{\epsilon} \mathbf{B}_{\epsilon}\right)^{-1} \mathbf{B}_{\epsilon}^{T} \mathbf{P}_{\epsilon} \mathbf{A}_{\epsilon}
\end{aligned}
$$

with

$$
\mathbf{R}=\operatorname{diag}\left(\mathbf{R}_{1}, \ldots, \mathbf{R}_{N}\right) .
$$

It is to be noted from the expression of the DARRE that the inversion of the partitioned matrices $\mathbf{R}_{\epsilon}+\mathbf{B}_{\epsilon}^{T} \mathbf{P}_{\epsilon} \mathbf{B}_{\epsilon}$ will produce a lot of terms and make the DARRE approach computationally very involved, even though one is faced with the reduced-order numerical problem. This problem is resolved by using a bilinear transformation to transform the discrete-time Riccati equations into a continuous-time algebraic Riccati equation with an equivalent correlation (Shen and Gajic, 1990). The differential game Riccati matrices satisfy the Singularly perturbed and Weakly coupled continuous in time Algebraic Regulator Riccati Equation (SWARRE) (Gajic and Shen, 1993; Sagara et al., 2008; Mukaidani, 2009):

$$
\begin{aligned}
& \boldsymbol{\Omega}_{i}\left(\mathbf{P}_{1 \epsilon}, \ldots, \mathbf{P}_{i \epsilon}, \ldots, \mathbf{P}_{N \epsilon}\right) \\
& =\mathbf{P}_{i \epsilon}\left(\mathbf{A}_{\epsilon}-\sum_{\substack{j=1 \\
j \neq i}}^{N} \mathbf{S}_{j \epsilon} \mathbf{P}_{j \epsilon}\right) \\
& +\left(\mathbf{A}_{\epsilon}-\sum_{\substack{j=1 \\
j \neq i}}^{N} \mathbf{S}_{j \epsilon} \mathbf{P}_{j \epsilon}\right)^{T} \mathbf{P}_{i \epsilon}-\mathbf{P}_{i \epsilon} \mathbf{S}_{i \epsilon} \mathbf{P}_{i \epsilon} \\
& +\sum_{\substack{j=1 \\
j \neq i}}^{N} \epsilon_{i j} \mathbf{P}_{j \epsilon} \mathbf{S}_{i j \epsilon} \mathbf{P}_{j \epsilon}+\mathbf{P}_{i \epsilon} \mathbf{M}_{i \epsilon} \mathbf{P}_{i \epsilon} \\
& +\mathbf{D}_{i \epsilon}^{T} \mathbf{D}_{i \epsilon}=0,
\end{aligned}
$$


where

$$
\begin{aligned}
\mathbf{S}_{i \epsilon} & =\mathbf{B}_{i \epsilon} \mathbf{R}_{i i}^{-1} \mathbf{B}_{i \epsilon}^{T}, \quad i=1, \ldots, N \\
\mathbf{S}_{i j} & =\mathbf{B}_{i \epsilon} \mathbf{R}_{j j}^{-1} \mathbf{R}_{i j} \mathbf{R}_{j j}^{-1} \mathbf{B}_{j \epsilon}^{T}, \quad i=1, \ldots, N \\
\mathbf{M}_{i \epsilon} & =\mathbf{W}_{\epsilon} \Theta_{w i \epsilon}^{-1} \mathbf{W}_{\epsilon}^{T}, \quad i=1, \ldots, N
\end{aligned}
$$

3.2. Auxiliary SWARRE. Substituting partitioned matrices $\mathbf{A}_{\epsilon}, \mathbf{S}_{i \epsilon}, \mathbf{S}_{i j \epsilon}, \mathbf{M}_{i \epsilon}, \mathbf{D}_{i \epsilon}$ and $\mathbf{P}_{i \epsilon}$ into the SWARRE (25), and letting $\epsilon_{w}=0$ and any $\epsilon_{s} \neq 0$, and then partitioning the SWARRE (25), we obtain the following reduced order (auxiliary) algebraic Riccati equation:

$$
\mathbf{P}_{i i} \mathbf{A}_{i i}+\mathbf{A}_{i i}^{T} \mathbf{P}_{i i}-\mathbf{P}_{i i}\left(\mathbf{S}_{i i}-\mathbf{M}_{i i}\right) \mathbf{P}_{i i}+\mathbf{D}_{i i}^{T} \mathbf{D}_{i i}=0,
$$

where $\mathbf{S}_{i i}=\mathbf{B}_{i i} \mathbf{R}_{i i}^{-1} \mathbf{B}_{i i}^{T}, \mathbf{M}_{i i}=\mathbf{W}_{i i} \Theta_{i i}^{-1} \mathbf{W}_{i i}^{T}$ and $\mathbf{P}_{i i}, i=1, \ldots, N$, is the zeroth-order approximation of $\mathbf{P}_{i \epsilon}$ when the weakly coupled parameter $\epsilon_{w}=0$. It should be noted from (26) that a unique positive semi-definite optimal solution $\mathbf{P}_{i \epsilon}^{*}$ exists if the following assumptions are taken into account.

Assumption 2. The triples $\mathbf{A}_{i i}, \mathbf{B}_{i i}$ and $\mathbf{D}_{i i}$, $i=1, \ldots, N$, are stabilizable and detectable.

Assumption 3. The auxiliary (26) has a positive semidefinite stabilizing solution such that $\widetilde{\mathbf{A}}=\mathbf{A}_{i i}-\mathbf{S}_{i i} \mathbf{P}_{i i}$ is stable.

Lemma 4. Under Assumption 3 there exists a small constant $\partial^{*}$ such that, for all $\widetilde{\epsilon}(t) \in\left(0, \partial^{*}\right)$, the SWARRE admits a positive definite solution $\mathbf{P}_{i \epsilon}^{*}$ represented as

$$
\begin{aligned}
\mathbf{P}_{i \epsilon} & =\mathbf{P}_{i \epsilon}^{*}=\mathbf{P}_{i}+O(\widetilde{\epsilon}(t)) \\
& =\operatorname{block} \operatorname{diag}\left(0 \cdots \mathbf{P}_{i i} \cdots 0\right)+O(\widetilde{\epsilon}(t))
\end{aligned}
$$

with $i=1, \ldots, N$ and $\tilde{\epsilon}(t)=\left|\sqrt{\epsilon_{w} \epsilon_{s}}\right|$.

Proof. This can be achieved by showing that the Jacobian the SWARRE is non-singular at $\widetilde{\epsilon}(t)=0$ and its neighbourhood $\epsilon(t) \rightarrow 0^{+}$.

Differentiating $\quad \Omega_{i}\left(\widetilde{\epsilon}(t), \mathbf{P}_{1 \epsilon}, \ldots, \mathbf{P}_{i \epsilon}, \ldots, \mathbf{P}_{N \epsilon}\right)$ with respect to the matrix $\mathbf{P}_{i \epsilon}$, we have

$$
\begin{aligned}
\mathbf{J}_{i i}= & \frac{\partial}{\partial \operatorname{vec} \mathbf{P}_{i \epsilon}} \operatorname{vec} \Omega_{i}\left(\widetilde{\epsilon}(t), \mathbf{P}_{1 \epsilon}, \ldots, \mathbf{P}_{N \epsilon}\right)^{T} \\
= & \Delta_{i i}^{T} \otimes I_{n_{i}}+I_{n_{i}} \otimes \Delta_{i i}^{T}, \\
\mathbf{J}_{i j}= & \frac{\partial}{\partial \operatorname{vec} \mathbf{P}_{i j}} \operatorname{vec} \Omega_{i}\left(\widetilde{\epsilon}(t), \mathbf{P}_{1 \epsilon}, \ldots, \mathbf{P}_{N \epsilon}\right)^{T} \\
= & -\left(\mathbf{S}_{j \epsilon} \mathbf{P}_{i \epsilon}-\widetilde{\epsilon}_{i j} \mathbf{S}_{i j \epsilon} \mathbf{P}_{j \epsilon}\right)^{T} \otimes I_{n_{i}} \\
& -I_{n_{i}} \otimes\left(\mathbf{S}_{j \epsilon} \mathbf{P}_{i \epsilon}-\widetilde{\epsilon}_{i j} \mathbf{S}_{i j \epsilon} \mathbf{P}_{j \epsilon}\right)^{T},
\end{aligned}
$$

where $i \neq j, j=1, \ldots, N$, and

$$
\Delta=\mathbf{A}_{\epsilon}-\sum_{\substack{j=1 \\ j \neq i}}^{N} \mathbf{S}_{j \epsilon} \mathbf{P}_{j \epsilon}+\mathbf{M}_{i \epsilon} \mathbf{P}_{i \epsilon} .
$$

Based on the fact that $\mathbf{S}_{j \epsilon} \mathbf{P}_{i \epsilon}=O(\widetilde{\epsilon}(t))$ for $i \neq$ $j$, the Jacobian of the SWARRE with $\widetilde{\epsilon}(t) \rightarrow 0^{+}$can be shown to be

$$
\begin{aligned}
& \hat{\mathbf{J}}=\operatorname{block} \operatorname{diag}\left(\Delta_{i 1}, \ldots, \Delta_{N N}\right), \\
& \mathbf{J}=\operatorname{block} \operatorname{diag}(\hat{\mathbf{J}}, \ldots, \hat{\mathbf{J}}) .
\end{aligned}
$$

Since the determinant of $\Delta_{i i}=\mathbf{A}_{i i}-\mathbf{S}_{i i} \mathbf{P}_{i i}+$ $\mathbf{M}_{i i} \mathbf{P}_{i i}$ with $\widetilde{\epsilon}(t)=0$ is non-zero by following assumption 3 for all $i=1, \ldots, N$, we get $\operatorname{det} \mathbf{J} \neq 0$, i.e., $\mathbf{J}$ is non-singular for $\widetilde{\epsilon}(t)=0$. As a consequence of the implicit function theorem coined by Jittorntrum (1978), $P_{i i}$ is a positive definite matrix at $\widetilde{\epsilon}(t)=0$, and for a sufficiently small parameter $\widetilde{\epsilon}(t) \in\left(0, \partial^{*}\right)$ we conclude that $\mathbf{P}_{i \epsilon}=\mathbf{P}_{i i}+O(\widetilde{\epsilon}(t))$ is also a positive definite solution.

The high-order Nash equilibrium strategy for signindefinite linear quadratic games is then obtained from the iterative solution given by

$$
\begin{aligned}
\mathbf{u}_{i}^{(k) *}(t)= & -\mathbf{R}_{i i}^{-1} \mathbf{B}_{i \epsilon}^{T} \mathbf{P}_{i \epsilon}^{(k)} \mathbf{x}(t), \\
& i=1, \ldots, N, \quad k=0,1,2, \ldots,
\end{aligned}
$$

where $\mathbf{u}_{i}^{*}=\mathbf{u}_{i}^{(k) *}+O(\widetilde{\epsilon})$ and $k$ is an iteration step. The optimal $u_{i}^{*}(t)$ is added to the power law $p_{i}(t+1)$ equation (15) such that $\sum_{i=1}^{N} p_{i}(t+1) \leq P_{\text {probe }}^{\text {tot }}$ Notation $N$ denotes the number of all users whose operations are managed by a single MRMC node and $P_{\text {probe }}^{\text {tot }}$ denotes the total channel probe power level which a node uses to upper bound greedy strategies.

\section{Decentralised power control algorithm}

Table 2 summarises the singularly perturbed and weakly coupled power control algorithm derived from useful results discussed in Sections 2 and 3 for each transmitting link of a multi-radio multi-channel backbone network. The algorithm considers message exchanges between transparent (i.e., same frequency channel) and immediate (i.e., in the vicinity) neighbours with low protocol overheads since no knowledge of the global topology is needed for its optimality. Moreover, a Carrier Sense Multiple Access with Collision Avoidance (CSMA/CA) medium access control scheme is utilised in order to schedule each transmission session randomly. In this scheme only actively transmitting links perform the power control algorithm in a randomly chosen time interval. Nodes cooperate with each other to forward packets from one node to another separated by many hops. The ultimate benefit derived from cooperation among the nodes is 
Table 2. Summarised power control algorithm.

\begin{tabular}{|c|c|}
\hline Step number & Action of each link and mathematical representation \\
\hline 0 & "At $t=t_{0}, \forall t_{0} \leq t \leq$ Packet transmission interval \\
\hline 1 & $\begin{array}{l}\text { Each node computes } \\
\varepsilon_{s} \text { from Eqns. (4), (5) and (7) } \\
\varepsilon_{w} \text { from Eqn. (14) } \\
\text { Nash equilibrium strategy for all links emanating from that node: } \\
u_{i}^{*}\left(t_{0}\right)=u_{i}^{(k) *}+\mathrm{O}(\tilde{\varepsilon}), \forall k \in\{0,1, \ldots\}\end{array}$ \\
\hline 2 & $\begin{array}{l}\text { Each node checks if the total power constraint is satisfied for all transmitting links originating } \\
\text { from it: } \\
\sum_{i=1}^{N} p_{i}\left(t_{0}\right) \leq p_{\text {probe }}^{\text {total }} \text {, where } \\
N \text { is the number of transmitting links connected to a node }\end{array}$ \\
\hline 3 & $\begin{array}{l}\text { Each link } i \text { sets its power to the initial arbitrary value: } \\
p_{i}\left(t_{0}\right)=p_{0}, \forall i \in\{1, \ldots, N\}\end{array}$ \\
\hline 4 & Otherwise \\
\hline 5 & $\begin{array}{l}\text { At } t \geq 0, \text { each link } i \text { adjusts the transmission power level according to the policy: } \\
p_{i}(t+1)=p_{i}(t)+u_{i}^{*}(t)\end{array}$ \\
\hline 6 & $\begin{array}{l}\text { Each link } i \text { sends pending DATA packets to its neighbouring destination at optimal power level } \\
\text { denoted as } p_{i}^{*}\end{array}$ \\
\hline 7 & If transmission, successful then link $i$ waits for the next packet arrival \\
\hline 8 & Else link $i$ returns to perform Step 2 \\
\hline
\end{tabular}

that a lower transmission power level can be selected and a higher spatial reuse can be achieved.

\section{Numerical examples}

The efficiency of the proposed model was studied using numerical examples computed in the MATLAB environment. MATLAB allows matrix manipulations and implementation of the queue-perturbed and weakly coupled power control algorithm in real Multi-Radio MultiChannel Wireless Networks. Furthermore, the MRMC WMNs considered could conveniently be modelled as a large-scale control system. In this case, decentralised architectures were exploited in order to minimise computational complexity of the large network while still achieving the desired study objectives. In particular, 5 to 50 wireless nodes were randomly placed in a $1200 \mathrm{~m} \times$ $1200 \mathrm{~m}$ region. Such a node placement scenario could allow possible multi-hop communication through cooperative forwarding of packets between randomly chosen source-destination pairs. Multi-hop communications ensured that a signal propagates over shorter wireless links between neighbouring nodes and, as a result, transmission power levels can significantly be reduced. Furthermore, effects of different node densities and interference on the convergence rate of the proposed power control could be studied numerically.

For simplicity, we assumed that each node had four Network Interface Cards (NICs) or radios installed and each radio was tuned to separate a non-overlapping Unified Channel Graph (UCG) as shown in Fig. 4. Such configurations were chosen in order to emulate weak coupling (a measure of a low interference level) among non- overlapping frequency channels and multiple queue perturbation between energy consumption and queue contents at a given transmission time (see Section 2). However, since radios on the same node happened to be closely seperated, their full duplex communication could cause inter-channel interference leading to packet drops and retransmissions at the expense of an extra energy consumption. Moreover, we utilised the ISM carrier frequency band of $2.427-2.472 \mathrm{GHz}$ with the bandwidth of $10 \mathrm{MHz}$ per frequency channel. Consequently, Fig. 4 illustrates a typical wireless MRMC network scenario with four nodes.

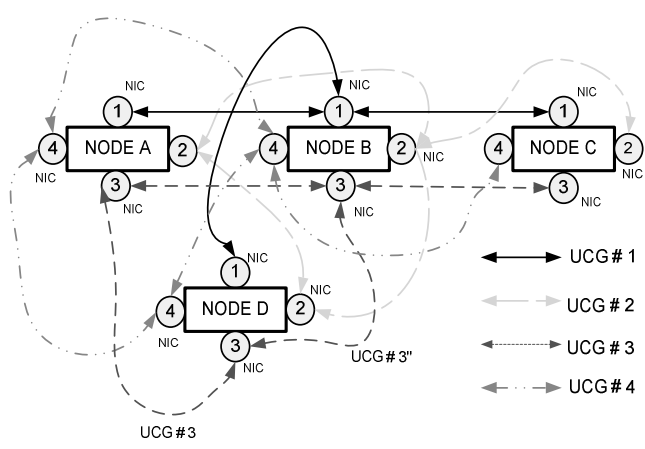

Fig. 4. Multi-radio multi-channel wireless communication network.

Each node has four NICs that can operate simultaneously in four disjoint channels (or UCGs). Node $A$ reaches Node $B$ and $D$ via one hop and it reaches $C$ via two hops (i.e., through Node $B$ ) or three hops (i.e., 
through Nodes $D$ and $B$ ). For example, NIC (radio) $B 1$ has transparent neighbours: $\{A 1, C 1, D 1\}$, radio $B 2$ has transparent neighbours: $\{A 2, C 2, D 2\}$ and radio $B 3$ has transparent neighbours: $\{A 3, C 3, D 3\}$. Radio $A 1$ can communicate with radio $B 1$ if tuned to UCG\#1 during the current timeslot and radio $B 1$ can relay the traffic of radio $A 1$ to radio $C 1$ if both are switched to $\mathrm{UCG} \# 1$ during the next timeslot. In order to focus our contribution, the discussion is limited to communication among transparent and immediate neighbours (decentralised model). Consequently, aware of the queue system perturbation problem and cross-channel interference coupling influence, each radio pair (user) optimizes transmission power levels while the link layer manages fairness in energy resource allocation (Olwal et al., 2009b).

5.1. Singular perturbation performance. Through a numerical example, an average transmission probability and a queue delay plotted against the packet arrival probability given a known level of the buffer size and transmission energy availability are obtained in Fig. 5. The reason for such performance metrics is to assess the effects of queue perturbation and delay on optimal power control in an MRMC node. The intuitive explanation is as follows: As packets arrive at the queues, the probability of transmitting a packet slightly increases but it is inversely proportional to the system perturbation. A highly perturbed queue system results in packet drops, and hence losses.

The slowly rising exponential delay behaviour versus packet arrivals under the influence of system perturbation suggests buffer overflows, and consequently undesirable queue delays. Figure 6 illustrates energy levels and the buffer size in order to have a lower bound transmission probability. The plotted levels are considered with different perturbations. For each value of the lower bound and a singular perturbation factor, the transmission energy level and the buffer size are determined such that the transmission probability should be no less than the lower bound transmission probability.

5.2. Parameter specifications. Given static backbone wireless nodes and allowing each multi-radio node to send the queue traffic to its target receivers (neighbours), the channel state coefficient matrices in (17) can be generated. In order to address a low computational complexity problem, the number of active neighbouring nodes was assumed to be at most five. The assumption is reasonable considering a typical deployment scenario of a backbone wireless mesh network. Moreover, due to space constraints regarding large dimensions of matrices, each node was taken to be having at least two radios: one for accessing the network and the other for routing backhaul traffic or, alternatively, both radios could be used at the backhaul for increasing the backhaul capacity. It should be


Fig. 5. Average transmission probability and delay vs. arrival probability in different singular perturbations. Buffer size $B=20$. Available energy levels for packet transmission $E=2$. Service probability $\varphi_{1}=1, \varphi_{2}=0.2$ and arrival probability $\phi=0.6$. Energy transition process $\lambda_{11}=0.3$ and $\lambda_{22}=0.5$. Probability distribution of the aggregated Markov chain $\Lambda_{1}, \overline{\mathcal{V}}_{1}=2 / 7$ and $\overline{\mathcal{V}}_{2}=5 / 7$.
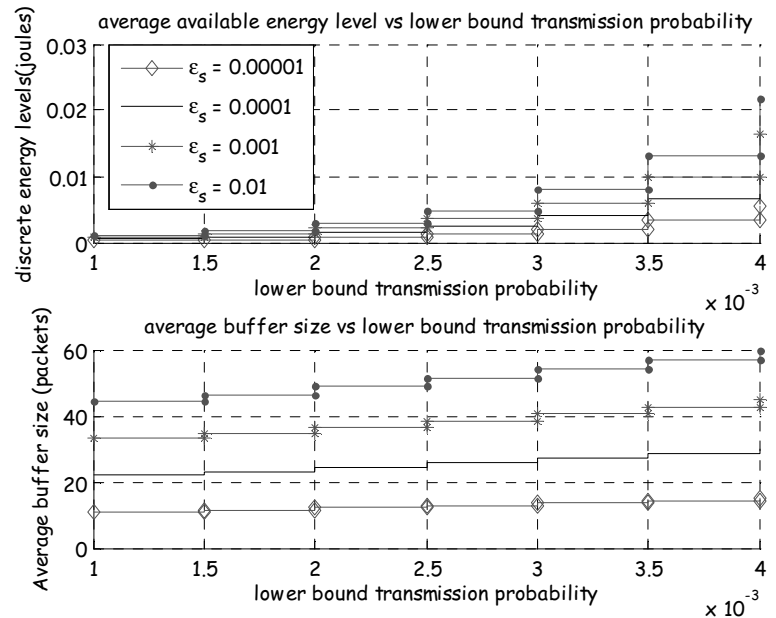

Fig. 6. Average energy and buffer levels vs. lower transmission probability in different singular perturbations. The average energy and the buffer size per network interface card needed to ensure a transmission probability of no less than the lower bound $p r_{l}, \phi=0.6, \varphi_{1}=1, \varphi_{2}=0.2$, $\lambda_{11}=0.3, \lambda_{22}=0.4, \lambda_{33}=\cdots=\lambda_{E}=0.1$ and $E=1$. Probability distribution of the aggregated Markov chain $\Lambda_{1}, \overline{\mathcal{V}}_{1}=\cdots=\overline{\mathcal{V}}_{E}=1 / 7$.

noted that such assumptions could still allow us to investigate effects of singular perturbation and weak coupling (in MRMC nodes) behaviours on the adjustment of the trans- 
mission power levels. Thus, other simulation parameters were numerically specified as follows:

$$
\begin{aligned}
& B_{11}=\left[\begin{array}{llll}
0.1 & 0.59 & 0 & 1
\end{array}\right]^{T}, \quad B_{11}=\left[\begin{array}{llll}
0 & 0.001 & 0 & 0.002
\end{array}\right]^{T}, \\
& B_{21}=\left[\begin{array}{lllll}
0 & 0.059 & 0 & 0.095
\end{array}\right]^{T}, \quad B_{22}=\left[\begin{array}{llll}
0.01 & 4 & 0 & 0.002
\end{array}\right]^{T},
\end{aligned}
$$

$$
\begin{aligned}
\Theta_{i i} & =\operatorname{diag}(0.90 .90 .90 .9), \\
\Theta_{1} & =\text { block diag }\left(\Theta_{i i} \epsilon_{w}^{-1} I_{4 \times 4}\right), \\
\mathbf{D}_{1} & =\text { block diag }\left(0.5 I_{4 \times 4} O_{4 \times 4}\right), \\
\mathbf{D}_{2} & =\text { block diag }\left(O_{4 \times 4} 0.5 I_{4 \times 4}\right), \\
\mathbf{R}_{12} & =\mathbf{R}_{21}=0.1 \\
\epsilon_{w} & =0.01, \quad \epsilon_{s}=0.001
\end{aligned}
$$

The above parameters were used to evaluate the performance of the proposed power control method in terms of stability and the rate of convergence. For a given node density, each transmitter-receiver pair (user) at a given transmission time $t$ was assumed to communicate its pending packets using an initially selected transmission power level, $p_{0}$. The stability and convergence of analytical results derived in Section 3.2 (i.e., the auxilliary SWARRE) and using the power control algorithm in Section 4 were then recorded. The simulation time was assumed long enough (i.e., 60 seconds) for the output statistics to stabilize. Each datum point obtained was a result of the average of all data points observed over all node densities tried. The reason behind this decision was to show that, regardless of the network size, the decentralised approach does not degrade the power control performance of the network.

5.3. System stabilizing solutions. The SWARRE solution $\mathbf{P}_{i \epsilon}^{(8) *}$ and the corresponding feedback matrices are $\mathbf{F}_{i \epsilon}^{(8) *}$ given as in Tables 3 and 4, respectively. Close examination of the $\mathbf{P}_{i \epsilon}^{(8) *}$ matrices reveals an $8 \times 8$ positive semi-definite stabilizing solution to the system matrices $\mathbf{A}_{i i}, \mathbf{B}_{i i}$ and $\mathbf{D}_{i i}$ in accordance with Assumption 2 such that the stability condition of Assumption 3 is satisfied. That is, the matrix $\mathbf{A}_{i i}-\mathbf{S}_{i i} \mathbf{P}_{i i}$ has eigenvalues strictly negative real parts.

The high-order approximate soft-constrained Nash strategies (27) are given by $\mathbf{u}_{i}^{(8) *}(t)=-\mathbf{F}_{i \epsilon}^{(8) *} \mathbf{x}(t)$, $i=1,2$, with $\mathbf{F}_{1 \epsilon}^{(8) *}$ and $\mathbf{F}_{2 \epsilon}^{(8) *}$ also given numerically from the SWARREs as shown in Table 4.

5.4. Objective function performance. The high-order soft constrained Nash strategy (approximation) and the zero-order (optimal) cost functions are respectively com- puted as follows:

$$
\begin{aligned}
J_{i, \text { approx }}^{*} & =J_{i}\left(\mathbf{u}_{1}^{(8) *}, \ldots, \mathbf{u}_{N}^{(8) *}, \mathbf{x}(0)\right) \\
& =\mathbf{x}(0)^{T} \mathbf{P}_{i \epsilon}^{(8) *} \mathbf{x}(0), \\
J_{i, \text { opt }}^{*} & =J_{i}\left(\mathbf{u}_{1}^{*}, \ldots, \mathbf{u}_{N}^{*}, \mathbf{x}(0)\right)=\mathbf{x}(0)^{T} \mathbf{P}_{i \epsilon}^{(8) *} \mathbf{x}(0),
\end{aligned}
$$

where $i=1,2$ for $N=2$. The initial state vector $\mathbf{x}(0)$ is

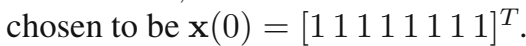

The cost function (27) and the degradation for the subsystem 1 are given in Table 5. The table illustrates that the cost function deviation at the eighth iteration yields an accuracy of

$$
\left\|J_{1, \text { opt }}^{*}-J_{1, \text { approx }}^{*}\right\|<7.294787 e^{-11} .
$$

This observation confirms that the proposed model converges practically to the exact solution. Furthermore, at the systems convergence point it can be shown that the cost functional to the SPWC ratio is very large, i.e., $\zeta_{i} \geq$ $7.294787 e^{+247}$, for all $i=1,2, \ldots, N$, where

$$
\zeta_{i}=\frac{\left|J_{1, \text { opt }}^{*}-J_{1, \text { approx }}^{*}\right|}{\tilde{\epsilon}^{2^{k}+1}}
$$

is the cost functional to SPWC ratio.

Figure 7 illustrates how the system cost functions converges exponentially fast. This fast response implies that the SWARRE solution can be obtained relatively fast if both system perturbation and inter channel interference information can be known. The wireless MRMC system states can be fast tracked to steady state solutions. The driving input control signal for power control is given by Fig. 8. For the dual radio system from Fig. 4, the input control signal convergences rapidly as shown in Fig. 8, and therefore the total CPU time can be saved significantly.

\section{Conclusion}

In this paper, SPWC model based power control in backbone wireless networks with MRMC configuration was discussed. First, the power control problem was assumed to be dependent on the queue perturbations due to the transmission energy level and buffer sizes. Consequently, a sufficiently small singular perturbation parameter was derived from the first-order Taylor series of the probability distribution of the two dimensional Markov chain. Second, interference across disjoint frequency channels was estimated via the geometry of signal radiation. The overlapping region between two separate channels was termed the estimate of the sufficiently small weak coupling parameter $\epsilon_{w}$. Third, differential Nash games were used to determine the optimal power control signal of each wireless link. The simulation results for a numerical example 
Table 3. Numerical values for the decomposed SWARRE.

\begin{tabular}{|l||c|c|c|c|c|c|c|c|}
\hline$P_{1 \epsilon}^{(8) *}$ & 5.6703 & 0.0408 & -0.1739 & -0.0155 & 0.0062 & 0.0004 & 0.0038 & 0.0003 \\
& 0.0408 & 0.5431 & 0.0299 & -0.0147 & -0.0004 & 0.0007 & -0.0003 & 0.0004 \\
& -0.1739 & 0.0299 & 2.4611 & 0.0301 & -0.0141 & -0.0008 & -0.0007 & 0.0002 \\
& -0.0155 & -0.0147 & 0.0301 & 0.1302 & -0.0002 & -0.0003 & -0.0001 & -0.0001 \\
& 0.0062 & -0.0004 & -0.0141 & -0.0002 & 0.2368 & 0.0054 & -0.0027 & 0.0001 \\
& 0.0004 & 0.0007 & -0.0008 & -0.0003 & 0.0054 & 0.0212 & -0.0001 & -0.0002 \\
& 0.0038 & -0.0003 & -0.0007 & -0.0001 & -0.0027 & -0.0001 & 0.2488 & 0.0031 \\
& 0.0003 & 0.0004 & 0.0002 & -0.0001 & 0.0001 & -0.0002 & 0.0031 & 0.0132 \\
\hline$P_{2 \epsilon}^{(8) *}$ & 2.3676 & 0.0537 & -0.0267 & 0.0013 & 0.0062 & 0.0004 & 0.0038 & 0.0003 \\
& 0.0537 & 0.2116 & -0.0012 & -0.0022 & -0.0004 & 0.0007 & -0.0003 & 0.0004 \\
& -0.0267 & -0.0012 & 2.4880 & 0.0314 & -0.0141 & -0.0008 & -0.0007 & 0.0002 \\
& 0.0013 & -0.0022 & 0.0314 & 0.1321 & -0.0002 & -0.0003 & -0.0001 & -0.0001 \\
& 0.0062 & -0.0004 & -0.0141 & -0.0002 & 0.2368 & 0.0054 & -0.0027 & 0.0001 \\
& 0.0004 & 0.0007 & -0.0008 & -0.0003 & 0.0054 & 0.0212 & -0.0001 & -0.0002 \\
& 0.0038 & -0.0003 & -0.0007 & -0.0001 & -0.0027 & -0.0001 & 0.2488 & 0.0031 \\
& 0.0003 & 0.0004 & 0.0002 & -0.0001 & 0.0001 & -0.0002 & 0.0031 & 0.0132 \\
\hline
\end{tabular}

Table 4. Near optimal (approx) regulator feedback values.

\begin{tabular}{|l||c|c|c|c|c|c|c|c|}
\hline$F_{1 \epsilon}^{(8) *}$ & -0.5755 & -0.3086 & 0.0303 & -0.1200 & -0.0002 & -0.0003 & -0.0001 & -0.0003 \\
\hline$F_{2 \epsilon}^{(8) *}$ & -0.2386 & -0.8470 & 0.0049 & 0.0088 & 0.0014 & -0.0029 & 0.0013 & -0.0016 \\
\hline
\end{tabular}

Table 5. Cost function and degradation performance.

\begin{tabular}{|c|c|c|c|c|}
\hline$k$ & $J_{1, \mathrm{opt}}^{*}$ & $J_{1, \text { approx }}^{(k) *}$ & $\left|J_{1, \text { opt }}^{*}-J_{1, \text { approx }}^{(k) *}\right|$ & $\zeta_{1}$ \\
\hline \hline 1 & $2.079347 \mathrm{e}+001$ & $2.082221 \mathrm{e}+001$ & $2.874104 \mathrm{e}-002$ & $2.874104 \mathrm{e}+001$ \\
2 & $2.079347 \mathrm{e}+001$ & $2.079944 \mathrm{e}+001$ & $5.971379 \mathrm{e}-003$ & $5.971379 \mathrm{e}+002$ \\
3 & $2.079347 \mathrm{e}+001$ & $2.079371 \mathrm{e}+001$ & $2.336666 \mathrm{e}-004$ & $2.336666 \mathrm{e}+005$ \\
4 & $2.079347 \mathrm{e}+001$ & $2.079348 \mathrm{e}+001$ & $2.324894 \mathrm{e}-006$ & $2.324894 \mathrm{e}+011$ \\
5 & $2.079347 \mathrm{e}+001$ & $2.079347 \mathrm{e}+001$ & $6.377017 \mathrm{e}-008$ & $6.377017 \mathrm{e}+025$ \\
6 & $2.079347 \mathrm{e}+001$ & $2.079347 \mathrm{e}+001$ & $1.048373 \mathrm{e}-008$ & $1.048373 \mathrm{e}+121$ \\
7 & $2.079347 \mathrm{e}+001$ & $2.079347 \mathrm{e}+001$ & $1.048373 \mathrm{e}-008$ & $1.048373 \mathrm{e}+121$ \\
8 & $2.079347 \mathrm{e}+001$ & $2.079347 \mathrm{e}+001$ & $7.294787 \mathrm{e}-011$ & $7.294787 \mathrm{e}+246$ \\
\hline
\end{tabular}

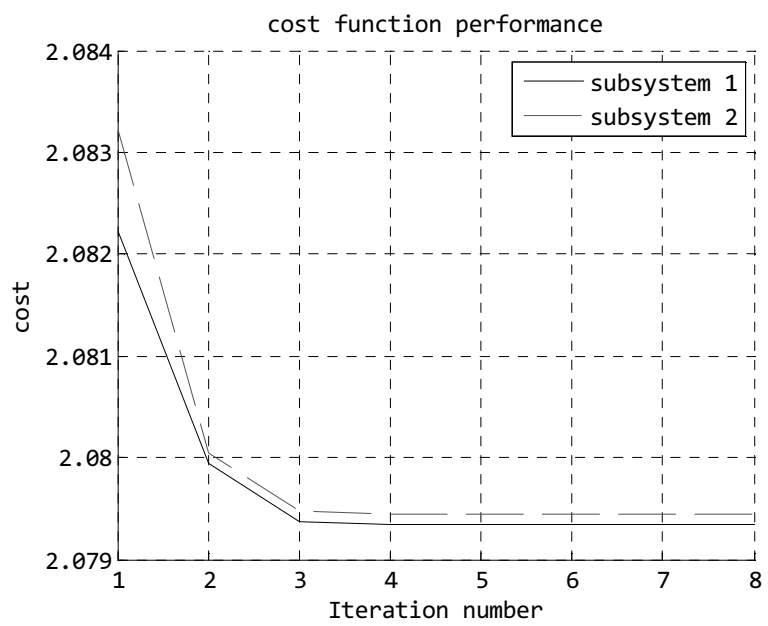

Fig. 7. Cost function performance.

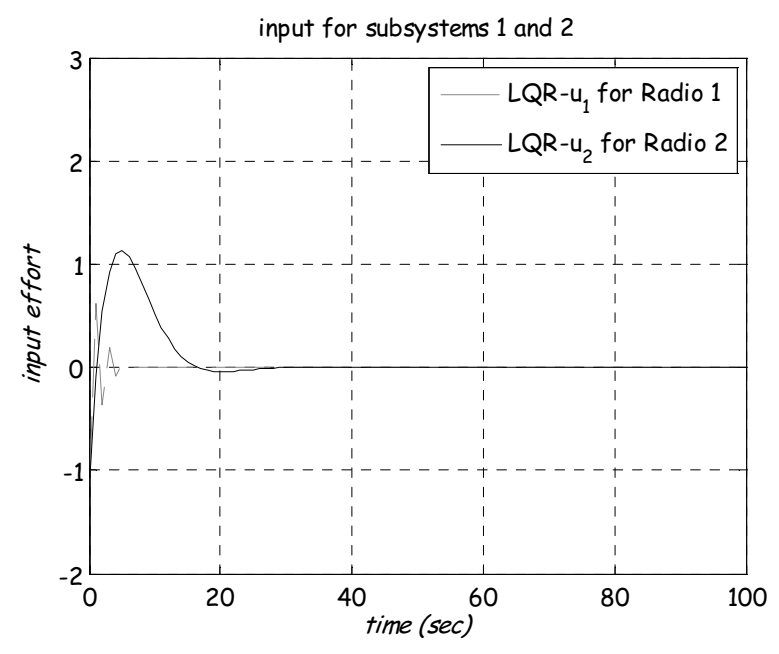

Fig. 8. Control input signal plotted against time [s]. 
showed performance improvement in terms of the convergence of the proposed power control method and the efficacy of the analytical model to decentralised backbone wireless networks.

The benefits achieved in this study include the characterization of the multi-radio multi-channel node network in terms of the singular perturbation and weak coupling behaviours that inherently cause energy consumption and yet have not been considered in the recent research endeavours. In addition, an improved power control performance was noted regardless of the network scale and density. However, the constraint of the model is that, as the dimension of MRMC configuration increases, the computation complexity consumes the CPU energy. In an attempt to overcome this constraint, this work demonstrated exponential convergence rates of power control. It should also be noted that this approach modelled a weakly coupled system using omni-directional radiation patterns. This was to mimic a mesh connectivity (i.e., redundancy) advantage as well as a worst case spatial reuse situation in backbone networks.

In practice, directional antennas with high gains and narrow beamwidths are currently being employed in backhaul mesh networks. However, switching and beam steering mechanisms implemented with directional antennas do come with high cost of latency in the network (Ramamurthi et al., 2008). Moreover, our approach would still apply in this set-up to resolve energy consumption problems stemming from multiple queues and undesired antenna side lobes. The method is also applicable in several commercially available wireless devices. Possible standards include IEEE 802.11a,b,g/n, IEEE 802.16 WiMAX, LTE and their heterogeneous versions in which MIMO techniques have been exploited in order to improve backhaul network capacity. Future work will demonstrate the effects of this new power control approach and routing on energy efficiency and network capacity.

\section{Acknowledgment}

The authors wish to thank the CSIR Meraka Institute and the Tshwane University of Technology for research grants. Appreciation goes to anonymous reviewers whose useful inputs helped to improve the quality of the paper.

\section{References}

Adisehu, H. and Parulkar, G. and Varghes, G. (1996). A reliable and scalable striping protocol, IEEE Signal Communication (SIGCOMM) 43(1):123-134.

Arora, A. and Krunz, M. (2007). Power controlled MAC for ad hoc networks with directional antennas, Elsevier Ad Hoc Networks 5(2): 145-161.

Avrachenkov, K.E. (1999). Analytic Perturbation Theory and Its Applications, Ph.D. thesis, University of South Australia, Adelaide.
Bruno, R., Conti, M. and Gregori, E. (2005). Mesh networks: Commodity multi-hop ad hoc networks, IEEE Communications Magazine 43(3): 123-134.

Chen, L., Zhang, Q., Li, M. and Jia, W. (2007). Joint topology control and routing in IEEE 802.11 based multiradio multichannel mesh networks, IEEE Transactions on Vehicular Technology 56(5): 3123-3136.

Chydziński, A. and Chróst, L. (2011). Analysis of AQM queues with queue size based packet dropping, International Journal of Applied Mathematics and Computer Science 21(3): 567-577, DOI:10.2478/v10006-011-0045-7.

Delebecque, F. and Quadrat, J. (1981). Optimal control of Markov chains admitting strong and weak interactions, $A u$ tomatica 17(2): 281-296.

El-Azouzi, R. and Altman, E. (2003). A queuing analysis of packet dropping over a wireless link with retransmissions, in M. Conti, S. Giordano, E. Gregori and S. Olariu (Eds.), Personal Wireless Communications, Lecture Notes in Computer Science, Vol. 2775, Springer, Berlin, pp. 321333.

Engim Inc. (2004). Multiple Channel 802.11 Chipset, http: / / www. engim.com/ products_en $3000 . \mathrm{html}$.

Gajic, Z. and Shen, X. (1993). Parallel Algorithms for Optimal Control of Large Scale Linear Systems, Springer-Verlag, London.

Ishmael, J., Bury, S., Pezaros, D. and Race, N. (2008). Rural community wireless networks, IEEE Internet Computing Journal 12(4): 22-29.

Jittorntrum, K. (1978). An implicit function theorem, Journal of Optimization Theory and Applications 25(4):285-288.

Klues, K., Xing, G. and C. Lu, C. (2006). A unified architecture for flexible radio power management in wireless sensor networks, Technical Report WUCSE-2006-06, Washington University in St. Louis, MO.

Li, N. and Hou, J.C. (2004). FLSS: A fault-tolerant topology control algorithm for wireless networks, Proceedings of the IEEE MobiCom Conference, New York, NY, USA. pp. 275286.

Li, N. and Hou, J.C. (2005). Localized topology control algorithms for heterogeneous wireless networks, IEEE/ACM Transactions on Networks 13(6): 1-6.

Mukaidani, H. (2009). Soft-constrained stochastic Nash games for weakly coupled large-scale systems, Automatica $\mathbf{4 5}(1)$ : 1272-1279.

Olwal, T.O., van Wyk, B.J., Djouani, K., Hamam, Y., Siarry, P and Ntlatlapa, P. (2009a). Autonomous transmission power adaptation for multi-radio multi-channel wireless mesh networks, Proceedings of the Ad Hoc-Now 2009 Conference, Murcia, Spain, pp. 284-297.

Olwal, T.O., van Wyk, B.J., Djouani, K., Hamam, Y., Siarry, P and Ntlatlapa, P. (2009b). Interference-aware power control for multi-radio multi-channel wireless mesh networks, Proceedings of the IEEE Africon 2009 Conference, Nairobi, Kenya, pp. 1-6. 
Olwal, T.O., van Wyk, B.J., Djouani, K., Hamam, Y., Siarry, P and Ntlatlapa, P. (2009c). A multiple-state based power control for multi-radio multi-channel wireless mesh networks, World Academy of Science, Engineering and Technology: International Journal of Computer Science 4(1): 53-61.

Olwal, T.O. (2010). Decentralized Dynamic Power Control for Wireless Backbone Mesh Networks, Ph.D. thesis, University of Paris-Est, Creteil.

Ramamurthi, V., Reaz, A., Dixit, S., and Mukherjee, B. (2008). Link scheduling and power control in wireless mesh networks with directional antennas, Proceedings of the IEEE Communication Conference 2008, Beijing, China, pp. 4835-4839.

Sagara, M., Mukaidani, H. and Yamamoto, T. (2008). efficient numerical computations of soft constrained nash strategy for weakly coupled large-scale systems, Journal of Computers 3(9): 2-10.

Shen, X. and Gajic, Z. (1990). Optimal reduced solution of the weakly coupled discrete Riccati equation, IEEE Transactions on Automatic Control 35(10): 1160-1162.

Schweitzer, P.J. (1986). Perturbation series expansions for nearly completely-decomposable Markov chains in O.J. Boxma, J.W. Cohen and H.C. Tijms (Eds.), Teletraffic Analysis and Computer Performance Evaluation, Elsevier Science Publishers, Amsterdam, pp. 319-328.

Sheth, A. and Han, R. (2005). SHUSH: Reactive transmit power control for wireless MAC Protocols, Proceedings of the 1st IEEE International Conference on the Wireless Internet (WICON), Budapest, Hungary, pp. 18-25.

Sorooshyari, S. and Gajic, Z. (2008). Autonomous dynamic power control for wireless networks: User-centric and network-centric consideration, IEEE Transactions on Wireless Communication 7(3): 1004-1015.

Tseng, Y.-C., Wu, S.-L., Lin, C.-Y and Shen, J.-P. (2001). A multi-channel MAC protocol with power control for multihop ad hoc networks, Proceedings of the Distributed Computing Systems Workshop, Boston, MA, USA, pp. 419-424.

Wang, K., Chiasserini, C.F., Proakis, J.G. and Rao, R.R. (2006). Joint scheduling and power control supporting multicasting in wireless ad hoc networks, Elsevier Ad Hoc Networks 4: $532-546$.

Zhu, H., Lu, K and Li,M. (2008). Distributed topology control in multi-channel multi-radio mesh networks, Proceedings of the IEEE International Communication Conference, Beijing, China, pp. 2958-2962.

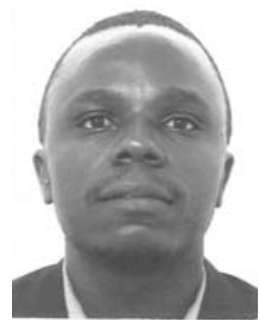

Thomas Otieno Olwal obtained his Ph.D. in computer science from the University of ParisEst (Creteil, France), and a D.Tech. in electrical engineering from the Tshwane University of Technology (South Africa) in 2010 and 2011, respectively. He is currently a senior scientific researcher at the Council for Scientific and Industrial Research (CSIR). He is also a member of the IEEE Communication and Computing Societies and a reviewer of IEEE Transactions journals. His research and development interests include radio resource allocation using behavioral ecology methods (foraging theory), singular perturbation, and weak coupling in future heterogeneous green and $\mathrm{cog}$ nitive radio communication networks. He has authored and co-authored four books as well as more than forty internationally recognised journal and conference papers.

Karim Djouani obtained his Ph.D. and D.Sc. in computer science from ISIEEE-Paris and the University of Paris 12 in 1994 and 2004, respectively. He is currently a professor at FSATI and a team member of SCTIC at the LISSI Lab, University of Paris Est-Creteil. His academic interests include soft computing, robotics and wireless networks. He is an IEEE member of the Communication and Computing Societies. He has authored and co-authored four books as well as more than 200 papers in journals and conference proceedings.

Okuthe P. Kogeda obtained his Ph.D. in computer science from the University of Western Cape (South Africa) in 2009. He is currently a senior lecturer and the head of the Postgraduate Section in the ICT Faculty at the Tshwane University of Technology. His research interests include biologically inspired modelling and simulation, Bayesian networks, and cellular networks. He has authored and co-authored more than 15 papers in journals and conference proceedings as well as book contributions.

Barend Jacobus van Wyk obtained his Ph.D. in electrical and information engineering from the University of Witwatersrand (South Africa) in 2003. He is currently a professor at FSATI and the Tshwane University of Technology (TUT), and the executive dean of the Faculty of Engineering and Building Environment (acting) at the TUT. His research interests include signal and image processing, pattern recognition, machine intelligence and control. He has authored and co-authored more than 200 papers in journals and conference proceedings as well as book contributions.
Received: 21 June 2011

Revised: 9 November 2011 